\title{
Pion-Nucleon Scattering in the Mandelstam Representation*
}

\author{
S. C. Frautschi \\ Department of Physics, University of California, Berkeley, California \\ AND \\ J. D. WALECKa $\ddagger$ \\ Institute of Theoretical Physics, Department of Physics, Stanford University, Stanford, California
}

(Received June 6, 1960)

\begin{abstract}
Using the analytic properties of partial wave scattering amplitudes, as derived from Mandelstam's representation, we have studied the $J=\frac{3}{2}, P$ state of the pion-nucleon system. The method used is covariant, it incorporates unitarity, and the effect of a possible pion-pion resonance has been investigated. Using the "single nucleon term" and the low-energy scattering properties of the "crossed states," we obtain a resonance in the $J=\frac{3}{2}, T=\frac{3}{2}$ pion-nucleon state without the aid of a cutoff. We have also investigated the scattering in the $T=\frac{1}{2}$ state. The pion-pion resonance appears to have only a very small effect in the $T=\frac{3}{2}$ state whereas in the $T=\frac{1}{2}$ state it increases the phase shift by a factor of 2 .

The resonance obtained in the $T=\frac{3}{2}$ state occurs at too low an energy. There are several factors which may account for this: We have not been able to include fully the contributions from crossed states, and we have not systematically included inelastic scattering.
\end{abstract}

\section{INTRODUCTION}

$T$ HE past few years have seen promising attempts to deal with strong-coupling theory by utilizing the analytic properties of scattering amplitudes. The work of Chew and Low ${ }^{1}$ on the static theory started this approach to the pion-nucleon scattering problem and enjoyed considerable success mainly because it embodied most of the principles that any correct theory must have. We have in mind here, in addition to the analytic properties, unitarity, conservation of isotopic spin, and crossing symmetry. Unfortunately, it was not possible to include Lorentz covariance among these in the static theory. Progress along the latter line was made by Chew, Goldberger, Low, and $\mathrm{Nambu}^{2}$ who utilized the fixed four-momentumtransfer dispersion relations to derive the successful results of the static theory and to include the leading kinematic corrections. While the latter authors had hopes of obtaining the position of the 3-3 resonance and the $s$-wave scattering lengths in principle, they were not able to do so in practice. Although their theory marked a great step forward in this field, the authors did not investigate the effect of a possible strong $\pi-\pi$ interaction nor was their projection of the partial wave amplitudes an exact one.

Mandelstam ${ }^{3}$ has recently proposed a two-dimensional representation of scattering amplitudes from which one can derive the analytic properties of partial

* Supported in part by the U. S. Air Force through the Air Force Office of Scientific Research.

† Supported by National Science Foundation Grant.

$¥$ National Science Foundation Postdoctoral Fellow.

1 G. F. Chew and F. E. Low, Phys. Rev. 101, 1570 (1956).

${ }^{2}$ G. F. Chew, M. L. Goldberger, F. E. Low, and Y. Nambu, Phys. Rev. 106, 1337 (1957). See also A. Finn, Phys. Rev. 119, 1786 (1960)

${ }^{3}$ S. Mandelstam, Phys. Rev. 112, 1344 (1958) and Phys. Rev. 115, 1741 and 1752 (1959). waves. $^{4,5}$ There is hope that by solving the $\pi-\pi$ scattering problem, which one can do within this framework, ${ }^{6}$ and working up through heavier mass states, one might finally be able to get a realistic hold on the strong coupling problem. That is, the goal is to calculate scattering amplitudes quantitatively in terms of the fundamental coupling constants of the theory and the masses of the particles involved. ${ }^{7}$ This program has had some successes, notably the justification of "polology" from which the same pion-nucleon coupling constant has been determined from several different physical processes. Frazer and Fulco $^{8}$ were also able to explain the isotopic vector part of the electromagnetic form factor of the nucleon by introducing a $\pi-\pi$ resonance in the $T=1, J=1$ state. There appear to be some theoretical arguments for this resonance ${ }^{6}$ and hopefully we will soon have some experimental evidence on this question.

In this paper we shall work on the second stage of the general program indicated above. Given the pion-nucleon coupling constant and a $\pi-\pi$ interaction, we investigate $\pi-N$ scattering.

We have collected the kinematic relations pertaining to pion-nucleon scattering in Sec. II. In Sec. III we discuss our choice of amplitude and Sec. IV contains the Mandelstam representation for this amplitude. Section $\mathrm{V}$ is concerned with a discussion of the program to be followed in practice and especially the replacement of known branch cuts by poles, and in Sec. VI we specialize to the $J=\frac{3}{2}$ amplitude and obtain an approxi-

${ }^{4}$ S. W. Macdowell, Phys. Rev. 116, 774 (1959).

${ }^{5}$ W. R. Frazer and J. R. Fulco, Phys. Rev. 119, 1420 (1960).

${ }^{6}$ G. F. Chew and S. Mandelstam, Phys. Rev. 119, 467 (1960) and Lawrence Radiation Laboratory Report UCRL-9126, March, 1960. C. F. Chew, S. Mandelstam, and H. P. Noyes, Phys. Rev. 119, 478 (1960).

${ }^{7}$ G. F. Chew, Lawrence Radiation Laboratory Report UCRL8670, January, 1959 (unpublished).

${ }^{8}$ W. R. Frazer and J. R. Fulco, Phys. Rev. 117, 1603 and 1609 (1960). 
mate solution for this amplitude including the "singlenucleon terms" and unitarity. "Long-range" contributions from crossed channels are considered in Sec. VII, and "short-range" and inelastic contributions are discussed in Sec. VIII. Section IX contains concluding remarks.

\section{KINEMATICS}

We first summarize the relevant formulas pertaining to the kinematics of pion-nucleon scattering. ${ }^{2}$ We take $p_{1}$ and $q_{1}$ to be the four-momenta of the incoming nucleon and meson, $p_{2}$ and $q_{2}$ to refer to the outgoing nucleon and meson, and $\alpha$ and $\beta$ to be the initial and final isotopic spin states of the pions (see Fig. 1). Then

$$
p_{1}+q_{1}=p_{2}+q_{2}
$$

by conservation of four-momentum. We shall refer to this process as channel I. We define the Lorentz scalars by

$$
\begin{aligned}
& s=-\left(p_{1}+q_{1}\right)^{2}, \\
& u=-\left(p_{2}-q_{1}\right)^{2}, \\
& t=-\left(q_{1}-q_{2}\right)^{2} .
\end{aligned}
$$

There is one relation between them,

$$
s+t+u=2 M^{2}+2 \mu^{2},
$$

which reduces the number of independent scalars to two. If $W$ is the total energy, $q$ the three-momentum, and $\theta$ the scattering angle, all in the center of mass, then

and therefore

$$
\begin{aligned}
& s=W^{2}, \\
& t=-2 q^{2}(1-\cos \theta),
\end{aligned}
$$

$$
u=2 M^{2}+2 \mu^{2}-W^{2}+2 q^{2}(1-\cos \theta) .
$$

We shall also need the relation

$$
q^{2}=\frac{\left[(W+M)^{2}-\mu^{2}\right]\left[(W-M)^{2}-\mu^{2}\right]}{4 W^{2}} .
$$

The $T$ matrix for this process is defined by

$$
\begin{aligned}
S_{f i}=\delta_{f i}-(2 \pi)^{4} i \delta^{4}\left(p_{2}+q_{2}-\right. & \left.p_{1}-q_{1}\right) \\
& \times\left(\frac{M^{2}}{4 E_{1} E_{2} \omega_{1} \omega_{2}}\right)^{\frac{1}{2}} \bar{u}_{2} T u_{1} .
\end{aligned}
$$

The $T$ matrix defined in this way $(\bar{u} u=1)$ is a Lorent $z$ scalar and can be written

$$
T=-A(s, u, t)+\frac{1}{2}\left(\boldsymbol{q}_{1}+\boldsymbol{q}_{2}\right) B(s, u, t),
$$

where

$$
\begin{aligned}
& A_{\beta \alpha}=\delta_{\beta \alpha} A^{+}+\frac{1}{2}\left[\tau_{\beta}, \tau_{\alpha}\right] A^{-}, \\
& B_{\beta \alpha}=\delta_{\beta \alpha} B^{+}+\frac{1}{2}\left[\tau_{\beta}, \tau_{\alpha}\right] B^{-} .
\end{aligned}
$$

The amplitudes $A^{ \pm}, B^{ \pm}$can be related to the isotopic
FIG. 1. Pion-nucleon interaction in channel I.

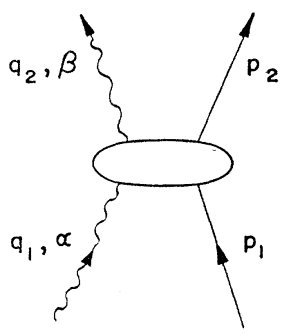

spin eigenamplitudes by

$$
\begin{aligned}
& A^{+}=\frac{1}{3}\left(A^{\frac{1}{2}}+2 A^{\frac{3}{2}}\right), \\
& A^{-}=\frac{1}{3}\left(A^{\frac{1}{2}}-A^{\frac{3}{2}}\right),
\end{aligned}
$$

with identical relations for the $B$ 's. In the center-of-mass system, the differential cross section can be written

$$
\frac{d \sigma}{d \Omega}=\sum_{\text {spins }}\left|\left(f\left|f_{1}+\frac{\left(\boldsymbol{\sigma} \cdot \mathbf{q}_{2}\right)\left(\boldsymbol{\sigma} \cdot \mathbf{q}_{1}\right)}{q_{2} q_{1}} f_{2}\right| i\right)\right|^{2},
$$

where the matrix element is taken between twocomponent spinors. The functions $f_{1}$ and $f_{2}$ are related to the phase shifts by

$$
\begin{aligned}
& f_{1}=\sum_{l=0}^{\infty} f_{l+} P_{l+1}{ }^{\prime}(x)-\sum_{l=2}^{\infty} f_{l-} P_{l-1}{ }^{\prime}(x), \\
& f_{2}=\sum_{l=1}^{\infty}\left(f_{l-}-f_{l+}\right) P_{l}^{\prime}(x)
\end{aligned}
$$

where

and

$$
x=\cos \Theta,
$$

$$
f_{l \pm}=e^{i \delta_{l \pm}} \sin \delta_{l \pm} / q,
$$

in the appropriate eigenstate of isotopic spin. These equations can be inverted by

$$
f_{l \pm}=\frac{1}{2} \int_{-1}^{1} d x\left[f_{1}(x) P_{l}(x)+f_{2}(x) P_{l \pm 1}(x)\right] .
$$

The functions $f_{1}$ and $f_{2}$ are related to $A$ and $B$ by

$$
\begin{aligned}
& f_{1}=\frac{(W+M)^{2}-\mu^{2}}{16 \pi W^{2}}[A+(W-M) B], \\
& f_{2}=\frac{(W-M)^{2}-\mu^{2}}{16 \pi W^{2}}[-A+(W+M) B],
\end{aligned}
$$

and conversely

$$
\begin{aligned}
& A=8 \pi W\left(\frac{W+M}{(W+M)^{2}-\mu^{2}} f_{1}-\frac{W-M}{(W-M)^{2}-\mu^{2}} f_{2}\right) \\
& B=8 \pi W\left(\frac{1}{(W+M)^{2}-\mu^{2}} f_{1}+\frac{1}{(W-M)^{2}-\mu^{2}} f_{2}\right) .
\end{aligned}
$$


Finally we can relate $f_{l \pm}$ to $A$ and $B$ by

$f_{l \pm}=\left(1 / 32 \pi W^{2}\right)\left\{\left[(W+M)^{2}-\mu^{2}\right]\right.$

$$
\begin{array}{r}
\times\left[A_{l}+(W-M) B_{l}\right]+\left[(W-M)^{2}-\mu^{2}\right] \\
\left.\times\left[-A_{l_{ \pm}}+(W+M) B_{l_{ \pm 1}}\right]\right\}
\end{array}
$$

where

$$
A_{l}(s)=\int_{-1}^{1} P_{l}(x) A(s, u, t) d x, \quad \text { etc. }
$$

Since $A_{l}$ and $B_{l}$ depend only on $W^{2}$, we have

$$
f_{l+}(W)=-f_{l+1-}(-W) .
$$

We shall refer to this as our "reflection principle."

It is a fundamental principle of the theory we will work with that the functions $A$ and $B$ are analytic functions of the variables $s, t$, and $u$ except for singularities associated with the three channels in Figs. 1, 2, and 3. That is, the boundary values of $A$ and $B$ describe the physical processes that can be obtained by any interchange of the legs of Fig. 1. The three physical channels obtained in this way are associated with nonoverlapping regions of the variables $s, t$, and $u$. The values of the functions $A$ and $B$ in the crossed pion-nucleon channel (Fig. 2) can be obtained from those of channel I by the use of crossing symmetry:

$$
\begin{aligned}
& A^{ \pm}(s, u, t)= \pm A^{ \pm}(u, s, t), \\
& B^{ \pm}(s, u, t)=\mp B^{ \pm}(u, s, t) .
\end{aligned}
$$

If the variables are such that channel III (Fig. 3) is physical, then

$$
\begin{aligned}
& t=4\left(\zeta^{2}+\mu^{2}\right)=4\left(p^{2}+M^{2}\right), \\
& s=-p^{2}-\zeta^{2}+2 p \zeta \cos \phi \\
& u=-p^{2}-\zeta^{2}-2 p \zeta \cos \phi,
\end{aligned}
$$

where $p$ is the nucleon momentum in the center-of-mass system of channel III, $\zeta$ is the pion momentum and

$$
y=\cos \phi=\mathbf{p}_{2} \cdot \zeta_{2} / p_{2} \zeta_{2} .
$$

In this case the amplitudes $A^{ \pm}$and $B^{ \pm}$are related to the eigenamplitudes of isotopic spin by ${ }^{8}$

$$
\begin{aligned}
& A^{+}=(1 / \sqrt{ } 6) A^{0}, \\
& A^{-}=\frac{1}{2} A^{1},
\end{aligned}
$$

etc. The amplitudes $A$ and $B$ can be written in terms of

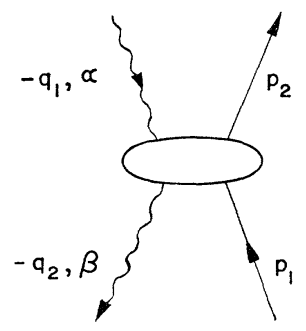

FIG. 2. Pion-nucleon interaction in channel II. partial wave helicity amplitudes ${ }^{9}$ by $^{8}$

$$
\begin{aligned}
A=- & \frac{8 \pi}{p^{2}}\left\{\sum_{J}\left(J+\frac{1}{2}\right)(p \zeta)^{J}\right. \\
& \left.\quad \times\left[f_{J+} P_{J}(y)-\frac{M}{[J(J+1)]^{\frac{1}{2}}} f_{J-} y P_{J}^{\prime}(y)\right]\right\},
\end{aligned}
$$

and

$$
B=8 \pi\left[\sum_{J} \frac{\left(J+\frac{1}{2}\right)}{[J(J+1)]^{\frac{1}{2}}}(p \zeta)^{J-1} f_{J-} P_{J}^{\prime}(y)\right],
$$

which are obtained by simple manipulations of the equations in reference (8). The reader is also referred to this work for the relation between the $f_{J_{ \pm}}$and the cross section in channel III.

\section{CHOICE OF AMPLITUDE}

Before proceeding to discuss the partial wave amplitudes as functions of a complex variable, we must first decide what variable to use and what kinematic factor to multiply the amplitudes by. The guiding

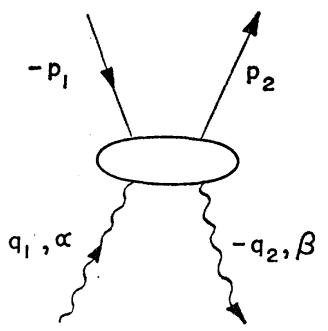

Fig. 3. Pion-nucleon interaction in channel III

principle in this decision will be to ensure that the main features of a correct theory are automatically maintained even when approximations are introduced.

First we shall explain our choice of variable. ${ }^{10}$ According to the Mandelstam representation the functions $A$ and $B$ are analytic functions of $W^{2}$ except for discontinuities determined by the "single-nucleon term" (Fig. 4) and physical scattering amplitudes in the three channels. The "single-nucleon" discontinuities are known functions of the pion-nucleon coupling constant. We shall also take low-energy scattering in crossed channels as given, and neglect high-energy scattering in crossed channels. The neglect of these high-energy scattering singularities in $A$ and $B$ has some chance of success because they are mostly far away in the complex plane, and the discontinuities across them are in most cases bounded by unitarity. Rather than work directly with $A$ and $B$, however, we shall use the partial wave amplitudes $f_{l_{ \pm}}[\mathrm{Eq} .(2.26)]$, because the simple unitarity conditions on the $f_{l_{ \pm}}$will be very important to us. It is evident from Eq. (2.26)

${ }^{9}$ M. Jacob and G. C. Wick, Ann. Phys. 7, 404 (1959).

${ }^{10}$ Some of these considerations have been introduced previously in references 4 and 5 . 
that $f_{l_{ \pm}}$contains singularities of type $\sqrt{ }\left(W^{2}\right)$ in the $W^{2}$ plane, in addition to the singularities in $A$ and $B$. The $\sqrt{ }\left(W^{2}\right)$ dependence is associated with nucleon spin; for example the factor $E \pm M$ in a Dirac spinor depends on $\sqrt{ }\left(W^{2}\right)$. Singularities of this type, which are not associated with single-nucleon terms or physical scattering amplitudes, are called "kinematic singularities." We wish to eliminate all kinematic singularities because the discontinuities across them are nowhere given or bounded by unitarity. ${ }^{11}$ This is easily accomplished by working in the $W$ rather than the $W^{2}$ plane. There may be other means of achieving the same end; the way we have chosen appears simple and straightforward to us.

An alternative possibility would be to use helicity amplitudes,,$^{9,4}$ which have no kinematic singularities in the $W^{2}$ plane. However, the helicity amplitude for a given $J$ and isotopic spin is a linear combination of the $l=J+\frac{1}{2}$ and $l=J-\frac{1}{2}$ states. This linear combination makes the unitarity conditions more complicated; for this reason we have not used helicity amplitudes.

Thus we consider the amplitude $\left(e^{i \delta} \sin \delta\right) / q$ as a function of $W$, using $\mathrm{Eq}$. (2.28) to give us a definition of the function in the left-hand $W$ plane. When the amplitude for any partial wave is written down [Eq. (2.26)], it contains an over-all factor of $W^{-2}$ from kinematics. The residue of this singularity is not known; therefore we eliminate it by multiplying the amplitude by $W^{2}$. The amplitude

$$
W^{2} e^{i \delta} \sin \delta / q
$$

now contains no kinematic singularities.

It is a general feature of quantum mechanics that any phase shift $\delta_{l}$ varies as $\delta_{l} \sim q^{2 l+1}$ at threshold. It can be shown that the amplitude for the $J, l=J-\frac{1}{2}$ state has $l\left(=J-\frac{1}{2}\right)$ wave thresholds in channels I (at $W=M+\mu)$ and II (at $W=M-\mu)$. Because of the reflection principle [Eq. (2.28)], the same amplitude has thresholds at negative $W(W=-M \pm \mu)$ which are related to the positive-energy $J, l=J+\frac{1}{2}$ wave in channels I and II. Thus our amplitude (3.1) for this state has zeroes for two positive values of $W$, near which it varies as $q^{2 l}$, and zeroes for two negative values of $W$, near which it varies as $q^{2 l+2}$.

We therefore introduce the following amplitude for the $J, l=J-\frac{1}{2}$ state:

$$
W^{2} e^{i \delta l+} \sin \delta_{l+} / q^{2 l+1}\left[(W+M)^{2}-\mu^{2}\right] .
$$

[The $J, l=J+\frac{1}{2}$ amplitude can be given in terms of this by using the reflection principle, (2.28).] The amplitude (3.2) has no kinematic singularities. It has built into it the correct threshold behavior; that is, our final answer will have the right threshold behavior

11 The self-consistent calculation of the discontinuities across these singularities, which would have to be carried out if they were not eliminated, would substantially increase the complexity of the problem.
FIG. 4. Diagrams of the "single-nucleon" terms.
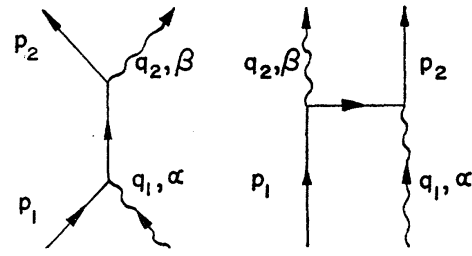

regardless of our approximation scheme. By dividing by the threshold factors, we have effectively made all subtractions which our knowledge of threshold behavior permits. Since $q^{-2} \sim W^{2}$ at $W \sim 0$ [Eq. (2.9)], it may appear that although division by $q^{2 l}$ removes the zeroes (of order $2 l$ ) at the physical thresholds on the right, it introduces the same number of zeroes at $W=0$. Appendix A contains an argument advanced by Mandelstam, ${ }^{12}$ which indicates that this will not be the case for us.

Dispersion integrals for the amplitude (3.2) converge very rapidly when $l>0$ due to the factors in the denominator. This good convergence was already implicitly present before we divided out the threshold zeroes, because the information on the threshold zeroes would allow several subtractions in the dispersion relations for amplitude (3.1). So our choice of kinematic factors in (3.2) has not really changed the maximum possible convergence of the dispersion relation for the amplitude. Likewise, we shall see in Sec. VI that the convergence of the " $N / D$ method" we use to solve the dispersion relation is in principle independent of kinematic factors such as $q^{-2 l}$ in the amplitude.

However, the kinematic factors are important in practice. The amplitude (3.2) falls off at least as fast as $W^{-(2 l+1)}$ as $W \rightarrow \infty$, because $q \sim W$ and $\left|e^{i \delta} \sin \delta\right| \leqslant 1$. In our method of solution the asymptotic behavior of a dispersion integral of form

$$
\int \frac{d W^{\prime} N\left(W^{\prime}\right)}{W^{\prime}-W}
$$

will essentially determine the asymptotic behavior of the amplitude. Although the amplitude (3.2) should fall off as $W^{-(2 l+1)}$ in principle, the contribution from the dispersion integral will fall off as $W^{-1}$ in practice because the cancellations needed to give better convergence cannot be maintained when unitarity is imposed in our calculation.

For the case $J=\frac{1}{2}$ the amplitude ${ }^{13}$

$$
g_{J=\frac{1}{2}}=W^{2} e^{i \delta} \sin \delta / q^{2}\left[(W+M)^{2}-\mu^{2}\right]
$$

is entirely satisfactory; it has no threshold zeroes or kinematic singularities and its correct asymptotic behavior is maintained even when unitarity is imposed.

\footnotetext{
${ }^{12} \mathrm{~S}$. Mandelstam (private communication).

${ }^{13}$ Calculations on this amplitude are now in progress.
} 
However, it is not possible to satisfy all these requirements for amplitudes with $J>\frac{1}{2}$, so some compromise must be made. The only example of $J>\frac{1}{2}$ we shall discuss here is $J=\frac{3}{2}$, which we need for the detailed calculations in Secs. VI-VIII. The amplitude we have used for this state is

$$
g_{1+}=W^{2} e^{i \delta_{1+}} \sin \delta_{1+} / q^{3}
$$

which appears to be a convenient, but not the only possible, choice. ${ }^{14}$ The $W^{-1}$ asymptotic behavior of (3.4) is automatically maintained when unitarity is imposed. A double zero has been reintroduced into (3.4) at the " $D$-wave threshold." This double zero is not maintained by our approximations, but the error introduced into the study of the $P$ wave should be small because the $D$ wave is not expected to have much reaction on the $P$ wave. In the end we shall check this point and find that the error indeed appears unimportant for the $P$ wave.

\section{THE MANDELSTAM REPRESENTATION}

According to Mandelstam's conjecture, the amplitudes $A^{ \pm}$and $B^{ \pm}$(referred to as $A^{i}$ with $i=1,2,3,4$ in this section for brevity) have a representation of the form ${ }^{3,15}$

$$
\begin{aligned}
A^{i}(s, u, t)= & \frac{R_{s}{ }^{i}}{M^{2}-s}+\frac{R_{u}^{i}}{M^{2}-u} \\
& +\frac{1}{\pi^{2}} \int_{(M+\mu)^{2}}^{\infty} d s^{\prime} \int_{(M+\mu)^{2}}^{\infty} d u^{\prime} \frac{\rho_{12}{ }^{i}\left(s^{\prime}, u^{\prime}\right)}{\left(s^{\prime}-s\right)\left(u^{\prime}-u\right)} \\
& +\frac{1}{\pi^{2}} \int_{(M+\mu)^{2}}^{\infty} d s^{\prime} \int_{4 \mu^{2}}^{\infty} d t^{\prime} \frac{\rho_{13}{ }^{i}\left(s^{\prime}, t^{\prime}\right)}{\left(s^{\prime}-s\right)\left(t^{\prime}-t\right)} \\
& +\frac{1}{\pi^{2}} \int_{(M+\mu)^{2}}^{\infty} d u^{\prime} \int_{2}^{\infty} d t^{\prime} \frac{\rho_{23}{ }^{i}\left(u^{\prime}, t^{\prime}\right)}{\left(u^{\prime}-u\right)\left(t^{\prime}-t\right)} .
\end{aligned}
$$

This can easily be rewritten as a one-dimensional representation at fixed $s$,

$$
\begin{array}{r}
A^{i}(s, u, t)=\frac{R_{s}{ }^{i}}{M^{2}-s}+\frac{R_{u}{ }^{i}}{M^{2}-u}+\frac{1}{\pi} \int_{4 \mu^{2}}^{\infty} \frac{d t^{\prime} A_{3}{ }^{i}\left(s, t^{\prime}\right)}{t^{\prime}-t} \\
+\frac{1}{\pi} \int_{(M+\mu)^{2}}^{\infty} \frac{d u^{\prime} A_{2}{ }^{i}\left(s, u^{\prime}\right)}{u^{\prime}-u}
\end{array}
$$

with the following representations of the weight

\footnotetext{
${ }^{14}$ Frazer and Fulco ${ }^{5}$ used an amplitude of the form of Eq. (3.2) to derive an effective-range relation for the $J=\frac{3}{2}, P$ state. They maintained the $W^{-3}$ falloff of their amplitude, in the course of the $N / D$ solution, by making two subtractions in $D$. By this means the $D$-wave threshold is treated correctly, but the subtractions introduce two experimental parameters (in addition to the masses and coupling constants) into the theory.

15 Subtraction terms have been omitted since they do not affect the arguments that follow.
}

functions :

$$
\begin{aligned}
& A_{3} i\left(s, t^{\prime}\right)=\frac{1}{\pi} \int_{(M+\mu) 2}^{\infty} \frac{d s^{\prime} \rho_{13}{ }^{i}\left(s^{\prime}, t^{\prime}\right)}{s^{\prime}-s} \\
& A_{2}^{i}\left(s, u^{\prime}\right)=\frac{1}{\pi} \int_{(M+\mu)^{2}}^{\infty} \frac{1}{\pi} \int_{-\infty}^{(M-\mu)^{2}-t^{\prime}} \frac{d s^{\prime} \rho_{23}{ }^{i}\left(s^{\prime}, t^{\prime}\right)}{s^{\prime}-s} \\
& s^{\prime}-s \frac{1}{\pi} \int_{-\infty}^{2 M^{2}-2 \mu^{2}-u^{\prime}} \\
& \frac{d s^{\prime} \rho_{23}{ }^{i}\left(s^{\prime}, u^{\prime}\right)}{s^{\prime}-s}
\end{aligned}
$$

The double spectral functions are real and do not extend up to the indicated boundaries except asymptotically. We have also kept $s+u+t=2 M^{2}+2 \mu^{2}$ for both the final and the dummy sets of variables.

From Eqs. (4.1)…(4.4), we can immediately deduce the analytic properties of the partial waves. We study these in the complex $W$ plane $\left(s=W^{2}\right)$ for reasons which have been discussed in the previous section. The quantities of interest to us are

$$
A_{l}^{i}(s)=\int_{-1}^{1} P_{l}(x) A^{i}(s, t(s, x), u(s, x)) d x
$$

since the partial waves are formed from linear combinations of these with coefficients that are simple functions of $W$. The only $x$ dependence in our fixed-s relation is in the denominators and therefore the partial waves are easily projected out:

$$
\begin{aligned}
A_{l}{ }^{i}(s)= & R_{s}{ }^{i} \int_{-1}^{1} \frac{P_{l}(x) d x}{M^{2}-s} \\
& +R_{u}^{i} \int_{-1}^{1} \frac{P_{l}(x) d x}{M^{2}-\left[2 M^{2}+2 \mu^{2}-s+2 q^{2}(1-x)\right]} \\
& +\frac{1}{\pi} \int_{4 \mu^{2}}^{\infty} d t^{\prime} A_{3}^{i}\left(s, t^{\prime}\right) \int_{-1}^{1} \frac{P_{l}(x) d x}{t^{\prime}+2 q^{2}(1-x)} \\
& +\frac{1}{\pi} \int_{(M+\mu)^{2}}^{\infty} d u^{\prime} A_{2}{ }^{i}\left(s, u^{\prime}\right) \\
& \times \int_{-1}^{1} \frac{P_{l}(x) d x}{u^{\prime}-\left[2 M^{2}+2 \mu^{2}-s+2 q^{2}(1-x)\right]}
\end{aligned}
$$

where we always have in mind that $q^{2}$ is a function of $s$ [Eq. (2.9)]. We are now in a position to ask what are the singularities of $A_{l}{ }^{i}(s)$. These will come from two sources. First, the denominators can vanish and thus give rise to branch cuts. Second, the functions $A_{3}{ }^{i}\left(s, t^{\prime}\right)$ and $A_{2}{ }^{i}\left(s, u^{\prime}\right)$ have singularities of their own, which can be determined from Eqs. (4.3), (4.4). We first discuss 
the latter type. Both $A_{3}$ and $A_{2}$ have a branch cut from $s=(M+\mu)^{2}$ to $\infty$, that is, for

$$
-\infty \leqslant W \leqslant-(M+\mu) \text { and }(M+\mu) \leqslant W \leqslant \infty .
$$

This branch cut corresponds to the physical region for pion-nucleon scattering in channel I and will be called the "physical cut." The second integral for each term appears to give a branch cut for negative $s$. Actually, this is not true and only arose from our partial fraction decomposition of $\left(u^{\prime}-u\right)^{-1}\left(t^{\prime}-t\right)^{-1}$. To see that in fact this singularity is fictitious we note that the second terms of $A_{2}$ and $A_{3}$ contribute to $A_{l}{ }^{i}$ as

$$
\begin{gathered}
-\frac{1}{\pi^{2}} \int_{4 \mu^{2}}^{\infty} d t^{\prime} \int_{-\infty}^{(M-\mu)^{2}-t^{\prime}} \frac{d s^{\prime} \rho_{23}\left(s^{\prime}, t^{\prime}\right)}{s^{\prime}-s} \int_{-1}^{1} \frac{P_{l}(x) d x}{t^{\prime}+2 q^{2}(1-x)} \\
-\frac{1}{\pi^{2}} \int_{(M+\mu)^{2}}^{\infty} d u^{\prime} \int_{-\infty}^{2 M^{2}-2 \mu^{2}-u^{\prime}} \frac{d s^{\prime} \rho_{23}\left(s^{\prime}, t^{\prime}\right)}{s^{\prime}-s} \\
\times \int_{-1}^{1} \frac{P_{l}(x) d x}{u^{\prime}-\left[2 M^{2}+2 \mu^{2}-s+2 q^{2}(1-x)\right]}
\end{gathered}
$$

If the orders of integration are interchanged in the $s^{\prime}, t^{\prime}, u^{\prime}$ integrations and the variable of integration then changed to $t^{\prime}=2 M^{2}+2 \mu^{2}-s^{\prime}-u^{\prime}$ in the second integral, one finds

$$
\begin{array}{r}
-\frac{1}{\pi^{2}} \int_{-\infty}^{(M-\mu)^{2}-4 \mu^{2}} d s^{\prime} \int_{4 \mu^{2}}^{(M-\mu)^{2}-s^{\prime}} \frac{d t^{\prime} \rho_{23}\left(s^{\prime}, t^{\prime}\right)}{s^{\prime}-s} \\
\quad \times \int_{-1}^{1} P_{l}(x) d x\left[\frac{1}{t^{\prime}+2 q^{2}(1-x)}\right] \\
\left.\quad+\frac{1}{s-2 q^{2}(1-x)-t^{\prime}-s^{\prime}}\right]
\end{array}
$$

and combining the two denominators gives an $s^{\prime}-s$ in the numerator.

We next state the results for the branch cuts arising from the vanishing of denominators in $A_{l}{ }^{i}(s)$, which we consider in three separate parts. The first part is the "single-nucleon term" (Fig. 4),

$$
\begin{aligned}
A_{l}{ }^{i}(s)^{N} & =R_{s}{ }^{i} \int_{-1}^{1} \frac{P_{l}(x) d x}{M^{2}-s} \\
& +R_{u}{ }^{i} \int_{-1}^{1} \frac{P_{l}(x) d x}{M^{2}-\left[2 M^{2}+2 \mu^{2}-s+2 q^{2}(1-x)\right]}
\end{aligned}
$$

which has the following properties:

1. The first term has poles at $W= \pm M$ if $l=0$.

2. The second term has a branch cut for

$$
\begin{aligned}
-\left(M^{2}+2 \mu^{2}\right)^{\frac{1}{2}} & \leqslant W \leqslant-\left(M^{2}-\mu^{2}\right) / M \\
\left(M^{2}-\mu^{2}\right) / M & \leqslant W \leqslant\left(M^{2}+2 \mu^{2}\right)^{\frac{1}{2}},
\end{aligned}
$$

as well as from 0 to $i \infty$ and 0 to $-i \infty$ (along the whole vertical axis).

The second part, which we call the "crossed $\pi-N$ cut," is associated with scattering in channel II:

$$
\begin{aligned}
A_{l^{i}}(s)^{\times}= & \frac{1}{\pi} \int_{(M+\mu)^{2}}^{\infty} d u^{\prime} A_{2}{ }^{i}\left(s, u^{\prime}\right) \\
& \times \int_{-1}^{1} \frac{P_{l}(x) d x}{u^{\prime}-\left[2 M^{2}+2 \mu^{2}-s+2 q^{2}(1-x)\right]}
\end{aligned}
$$

This term has a branch cut for

$$
-(M-\mu) \leqslant W \leqslant(M+\mu),
$$

and also from $-i \infty$ to $+i \infty$.

The third part, which we call the " $\pi-\pi$ cut," is associated with scattering in channel III:

$$
A_{l^{i}}(s)^{\pi \pi}=\frac{1}{\pi} \int_{4 \mu^{2}}^{\infty} d t^{\prime} A_{3}{ }^{i}\left(s, t^{\prime}\right) \int_{-1}^{1} \frac{P_{l}(x) d x}{t^{\prime}+2 q^{2}(1-x)} .
$$

This term has a cut along the circle

$$
|W|=\left(M^{2}-\mu^{2}\right)^{\frac{1}{2}}
$$

and also from $-i \infty$ to $+i \infty$.

Since our $W$ plane is really cut in two by the cut from $-i \infty$ to $+i \infty$, we are free to define the function as we like on the left (also inside the circle). We shall however use Eq. (4.6) for $A_{l}{ }^{i}$ to define the function on the left, and in the circle, for then we can relate the discontinuities across the cuts to the absorptive parts of scattering amplitudes. The cuts are summarized in Fig. 5.

The next step is to calculate the absorptive parts across the cuts in terms of $A_{2}{ }^{i}$ and $A_{3}{ }^{i}$. This is very easy

\section{W}

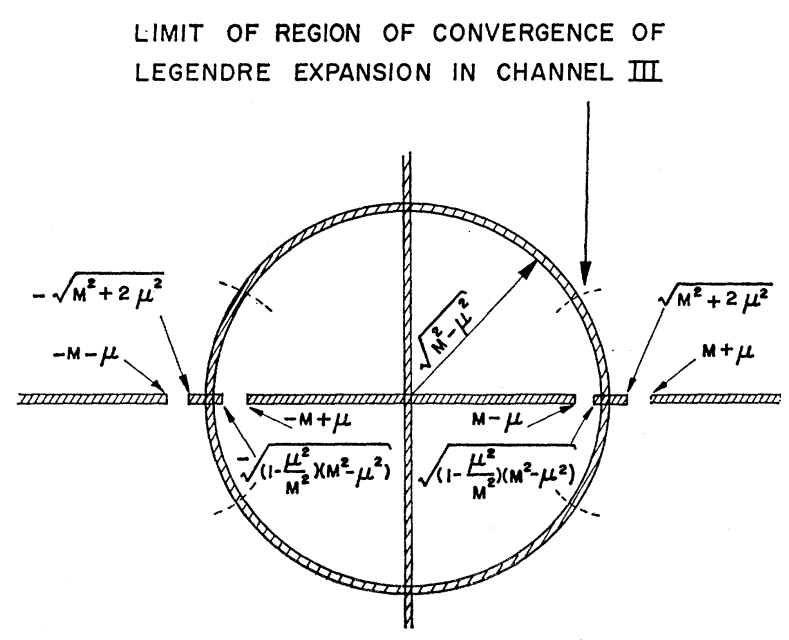

FIG. 5. Singularities of the partial wave pion-nucleon amplitudes in the $W$ plane. 
for the "dynamic singularities" (everything but the physical cut and its reflection) since one can treat the cuts as arising merely from the vanishing of the denominators in Eq. (4.2). This is perhaps not entirely obvious since on the cuts where $s$ is real the denominators of the second terms in Eqs. (4.3) and (4.4) can vanish and $A_{3}{ }^{i}$ and $A_{2}{ }^{i}$ will become complex and themselves have absorptive parts across the cuts. If we write, however,

$$
1 /\left(s^{\prime}-s\right)=\mathrm{P}\left[1 /\left(s^{\prime}-s\right)\right] \pm i \pi \delta\left(s^{\prime}-s\right)
$$

then, by Eq. (4.8), the term $i \pi \delta\left(s^{\prime}-s\right)$ will not contribute to the partial wave amplitude because its coefficient vanishes. We are therefore permitted to calculate the absorptive parts across the cuts entirely in terms of the vanishing of the denominators in Eq. (4.2), remembering that when $A_{2}{ }^{i}$ and $A_{3}{ }^{i}$ become complex (on the cuts indicated above) it is only their real part which will contribute.

Defining

\section{Crossed $\pi-N$ Cut}

$$
\operatorname{Abs} g(W)=\frac{1}{2 i}\left[g\left(W_{+}\right)-g\left(W_{-}\right)\right]
$$

where $W_{+}$refers to one side of the cut and $W_{-}$to the other side, we find

$$
\operatorname{Abs} A_{l}^{i}(s)^{\times}=( \pm) \int_{-1}^{1} P_{l}(x) A_{2}^{i}(s, u) d x
$$

The choice of sign is indicated in Fig. 6. It is located on the side of the cut which is taken first in calculating the absorptive part. Equation (4.13) can be more directly related to physical scattering by using crossing symmetry [Eq. (2.29) and see Eq. (4.20)], $A_{2}{ }^{i}(s, u)$ $=( \pm)_{i} A_{2}{ }^{i}(u, s)$. On the right-hand side, $u$ is now the energy in channel II, and $s$ is a momentum transfer. They are both physical in the region $0 \leq s \leq(M-\mu)^{2}$ but not in the region $s<0$. This can be seen in the following way. One can write the angle of scattering in channel $\mathrm{I}$ as a function of $s$ and $u$ :

$$
\begin{aligned}
x(s, u) & =1+\frac{2 s\left(2 M^{2}+2 \mu^{2}-s-u\right)}{s^{2}-2 s\left(M^{2}+\mu^{2}\right)+\left(M^{2}-\mu^{2}\right)^{2}} \\
& =-1+\frac{2\left[\left(M^{2}-\mu^{2}\right)^{2}-u s\right]}{s^{2}-2 s\left(M^{2}+\mu^{2}\right)+\left(M^{2}-\mu^{2}\right)^{2}} .
\end{aligned}
$$

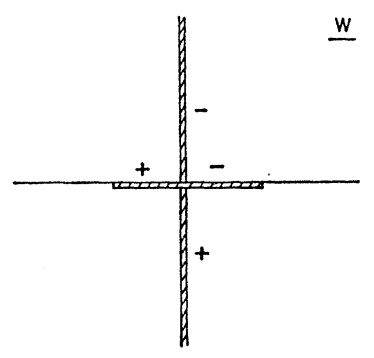

Frg. 6. Signs to be used in calculating the absorptive part of the crossed pion-nucleon term as given by Eq. (4.13).

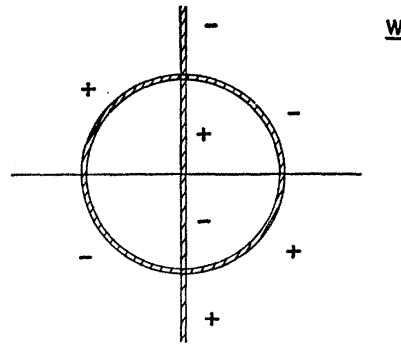

FIG. 7. Signs to be used in calculating the absorptive part of the crossed pion-pion term as given by Eq. (4.16).

Upon use of the crossing relations, this angle is transformed into the scattering angle in the channel II, $x^{\times}=x(u, s)$. For the values of $x$ used in the partial wave projection, $-1 \leq x \leq 1$, then it will also be true that $-1 \leq x^{\times} \leq 1$ and $u>(M+\mu)^{2}$ as long as $0 \leq s$ $\leq(M-\mu)^{2}$, but not when $s<0$. By changing variables from $x$ to $u$ in Eq. (4.13) one has for all real $s \leq(M-\mu)^{2}$,

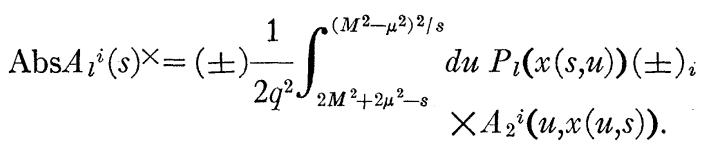

\section{2. $\pi-\pi \mathrm{Cut}$}

Proceeding in the same fashion one finds

$\operatorname{Abs} A_{l}^{i}(s)^{\pi \pi}$

$$
=( \pm)\left(\frac{-1}{2 q^{2}}\right) \int_{4 \mu^{2}}^{-4 q^{2}} d t P_{l}\left(1+\frac{t}{2 q^{2}}\right) A_{3}{ }^{i}(s, t),
$$

where again the signs are indicated in Fig. 7. In the special case where we are on the circle we have

$$
\begin{gathered}
-2 q^{2}=g(\Phi)=2\left[M^{2} \sin ^{2}(\Phi / 2)+\mu^{2} \cos ^{2}(\Phi / 2)\right] \\
\operatorname{Abs} A_{l^{i}}(s)^{\pi \pi} \quad W=\left(M^{2}-\mu^{2}\right)^{\frac{1}{2}} e^{i \Phi / 2} \\
=( \pm) \frac{1}{g(\Phi)} \int_{4 \mu^{2}}^{2 g(\Phi)} d t P_{l}\left(1-\frac{t}{g(\Phi)}\right) A_{3}{ }^{i}(t, s)
\end{gathered}
$$

In contrast to the $\pi-N$ crossed cut, there is no region on the $\pi-\pi$ cut that corresponds entirely to a physical process in channel III.

Finally, we proceed to show that the absorptive amplitudes $A_{2}$ and $A_{3}$ are real in certain regions where the denominators of the scattering amplitude $A[\mathrm{Eq}$. (4.1)] vanish. As a consequence, $A_{2}$ and $A_{3}$ can be identified with the imaginary part of the scattering amplitude in these regions.

\section{Crossed $\pi-N$ Cut}

If we let $s_{23}{ }^{\max }$ be the maximum value of $s$ in the region where the spectral function $\rho_{23}$ (Fig. 8) is nonvanishing, then for

$$
s_{23}^{\max } \leq s \leq(M+\mu)^{2},
$$


we find that both $A_{3}$ and $A_{2}$ are real. [The denominators of Eqs. (4.3), (4.4) cannot vanish and the double spectral functions are real.] If now $t<4 \mu^{2}$, then $1 /\left(t^{\prime}-t\right)$ is real and the only imaginary term comes from the vanishing of the denominator $1 /\left(u^{\prime}-u\right)$. We therefore have

$$
\begin{aligned}
& \operatorname{Im} A^{i}(s, u, t) \\
& =A_{2}{ }^{i}(s, u) \\
& =( \pm)_{i} \operatorname{Im} A^{i}(u, s, t)
\end{aligned} \quad\left\{\begin{array}{c}
s_{23}{ }^{\max } \leq s \leq(M+\mu)^{2} \\
t<4 \mu^{2} \\
u>(M+\mu)^{2} .
\end{array}\right.
$$

This region includes the low-energy crossed pion-nucleon scattering region.

\section{2. $\pi-\pi \mathrm{Cut}$}

Again if $s_{23}{ }^{\max } \leq s \leq(M+\mu)^{2}, A_{3}$ and $A_{2}$ are real. If $u<(M+\mu)^{2}$, then the only imaginary part comes from the vanishing of $t^{\prime}-t$ in Eq. (4.2) and

$$
\operatorname{Im} A^{i}(s, u, t)=A_{3}{ }^{i}(t, s)\left\{\begin{array}{c}
s_{23}{ }^{\max } \leq s \leq(M+\mu)^{2} \\
u<(M+\mu)^{2} \\
t>4 \mu^{2} .
\end{array}\right.
$$

This includes physical $\left(t \geq 4 M^{2}\right) \quad N+\bar{N} \rightarrow \pi+\pi$. It must be emphasized here that in this channel $t$ is the energy and $s$ is the momentum transfer. We need $A_{3}(t, s)$ on the circle, where $s$ is complex. We can use the Legendre expansion in this channel to give us a continuation in the complex momentum transfer over a limited region. (The validity of this expansion is discussed in Appendix C.)

We now, in principle, can calculate all the discontinuities on the crossed cuts in terms of scattering amplitudes for the crossed processes (although one will eventually have to study the double spectral functions in order to treat the regions where the Legendre expansion no longer converges). We proceed next to utilize these results.

\section{OUTLINE OF PROCEDURE AND USE OF POLES}

We are now in a position to outline our program. We shall take the scattering in the crossed states as given. This means we have a set of known discontinuities on the "dynamic singularities." We can then construct an amplitude that has these discontinuities and which is also unitary on the physical cut by using the $N / D$ method of solution due to Chew and Mandelstam. ${ }^{6}$ In principle, of course, the discontinuities on the dynamic singularities are not known independently of pion-nucleon scattering. What one would like to do in principle is to solve the $\pi-\pi$ scattering problem consistently in terms of itself ${ }^{6}$ and then to use this information as input and solve the pion-nucleon scattering problem consistently in terms of itself. In this way one would hope to generate all the scattering amplitudes in low-energy regions in terms of two coupling constants, one for the pion-pion interaction and one for the pion-nucleon interaction, and the masses of the particles involved. The first part of this program is under way. ${ }^{6}$
One difficulty with this part of the program is that there is so far no direct experimental evidence against which one can check the predictions for pion-pion scattering. We are of course more fortunate in the case of pionnucleon scattering where there is a rich fund of experimental data available. The solution of pion-nucleon scattering in terms of itself and of a given $\pi-\pi$ scattering is a difficult problem and one which we shall not actually carry out here although one would hope that it will be done sometime in the foreseeable future. What we shall do instead is to incorporate those features which we think the correct theory should have insofar as the dynamic singularities are concerned. We shall attempt to keep all the known symmetries of our theory, and see what amplitude we generate on the physical cut. (As of the present, we are only in a position to keep crossing symmetry approximately.) There are three terms contributing to the dynamic singularities. The first is the single-nucleon term (Fig. 4) which contains the pion-nucleon coupling constant and is a completely known function. We can thus take this term into account exactly. The importance of this term for the low-energy behavior of pion-nucleon scattering has been pointed out by Chew and Low. ${ }^{1}$ The second term contains the absorptive part of the process $N+\bar{N} \rightarrow \pi+\pi$. This amplitude has been discussed in detail by Frazer and Fulco. ${ }^{8}$ They show that if one includes a $J=1, T=1$ pion-pion resonance, then combining it with the single nucleon contribution to $N+\bar{N} \rightarrow \pi+\pi$ is enough to give agreement with the experimental results for the vector part of the magnetic form factor of the nucleon. We shall include in our calculation the contribution from this $\pi-\pi$ resonance and see what the effect is on pion-nucleon scattering. In including this amplitude, we are still working within the framework of the basic program outlined in the beginning of this section. The third term contributing to the dynamic singularities is the crossed $\pi-N$ cut. Here we have imposed crossing symmetry and related the contribution along the real axis to physical pion-nucleon scattering. Here one is really faced with the consistency problem which has not even been solved exactly in the static theory where crossing couples only the $P$ states at the same energy. We shall therefore

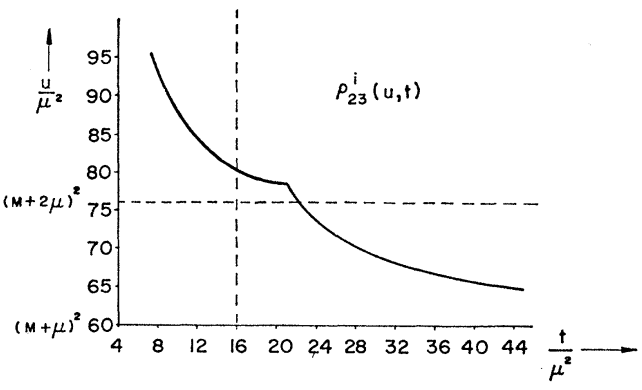

FIG. 8. Boundary curves of the Mandelstam two-dimensional spectral function. 
abandon the more fundamental course of calculating all the phase shifts without the aid of experimental data. Instead, we shall put in for the crossed cut the experimental observation that low-energy pion-nucleon scattering is dominated by the $3-3$ resonance. It will turn out, however, that this contribution is the smallest of the three discussed so far, so that our calculation can still be considered to be approximately within the framework of the more fundamental program.

Taking the discontinuities across the dynamic cuts as given, one can proceed by the $N / D$ method to solve for an amplitude which is unitary on the physical cut and has these discontinuities. The result is an integral equation which is rather complicated although it can be solved by straightforward methods. If the dynamic singularities were just poles instead of branch cuts, then one would have the simpler problem of solving a set of linear algebraic equations. We have decided to use the procedure of approximating the dynamic singularities by a set of poles and solving the resulting problem exactly. In addition to simplifying the problem, this procedure allows us to watch the behavior of our results as we vary the input information. For example, we can immediately spot the source of the difficulty if we are troubled by the appearance of ghosts or spurious bound states in our solution by the $N / D$ method.

We would like to be as precise as possible in replacing the cuts by poles and to do this we adopt the following criteria for these poles:

1. They must, of course, satisfy the general conjugation property

$$
g_{l}^{*}\left(W^{*}\right)=g_{l}(W) .
$$

[This property follows immediately from the RiemannSchwarz reflection principle and the fact that between the cuts on the real $W$ axis, $g_{l}(W)$ is a real function.]

2. They must be located at reasonable positions along the cuts so as to approximate the shape of the actual discontinuity.

3. They must have magnitudes and relative signs so as to approximate also those of the discontinuities.

4. The Cauchy integral around the poles must approximate the Cauchy integral around the dynamic cuts when it is evaluated in the physical region. The reason we ask this is that our final answer will be expressed in terms of integrals of these functions over the physical region.

5. Finally, we ask that our set of poles be as simple as possible, consistent with the above considerations.

The validity of approximating by poles can in principle be checked by solving the integral equation with the complete cuts in a given case. We have not done this but will later give some arguments on the validity of our procedure.

We shall treat the problem in two stages. We first treat the single-nucleon term and then add the contributions of the other dynamic singularities.

\section{SINGLE-NUCLEON TERM PLUS UNITARITY}

In this section we shall calculate the single-nucleon term and then generate from it a solution consistent with unitarity in channel I. The additional contributions provided by the crossed $\pi-N$ and $\pi-\pi$ channels, which complicate the problem considerably, will be added in the next section.

The single-nucleon diagrams are given in Fig. 4. For these diagrams, $A$ and $B$ are

$$
\begin{aligned}
& A=0, \\
& B^{\frac{3}{2}}=8 \pi g^{2} /\left(u-M^{2}\right), \\
& B^{\frac{1}{2}}=-4 \pi g^{2}\left[3 /\left(s-M^{2}\right)+1 /\left(u-M^{2}\right)\right],
\end{aligned}
$$

where $g^{2} \sim 15$ and the upper indices refer to isotopic spin. At this point we specialize to the $J=\frac{3}{2}$ state for the remainder of the paper. For the $J=\frac{3}{2}$ state we need the following partial wave projections:

$$
\begin{gathered}
B_{1^{\frac{3}{2}}}=\int_{-1}^{1} x d x B^{\frac{3}{2}}(x)=\frac{-4 \pi g^{2}}{q^{2}}\left[2-a \ln \frac{a+1}{a-1}\right], \\
B_{2^{\frac{3}{2}}}=\frac{-4 \pi g^{2}}{q^{2}}\left[-3 a+\left(\frac{3 a^{2}-1}{2}\right) \ln \frac{a+1}{a-1}\right],
\end{gathered}
$$

and

where

$$
a=\left(W^{2}-M^{2}-2 \mu^{2}\right) / 2 q^{2}-1 .
$$

We notice that for the single-nucleon term with $l \neq 0$,

$$
B l^{\frac{1}{2}}=-\frac{1}{2} B l^{\frac{3}{2}} .
$$

The $J=\frac{3}{2}$ amplitude,

$$
g_{1+}=W^{2} e^{i \delta_{1+}+} \sin \delta_{1+} / q^{3},
$$

is given by the general expression [Eqs. (2.26) and (2.20)]

$$
\begin{aligned}
g_{1+}= & \frac{1}{32 \pi q^{2}}\left\{\left[(W+M)^{2}-\mu^{2}\right]\left[A_{1}+(W-M) B_{1}\right]\right. \\
& \left.+\left[(W-M)^{2}-\mu^{2}\right]\left[-A_{2}+(W+M) B_{2}\right]\right\},
\end{aligned}
$$

which applies to either isotopic spin state. Substituting $(6.4) \cdots(6.7)$ into (6.9), we find the Born amplitudes

$$
\begin{aligned}
& g_{1+}\left(\begin{array}{l}
T=\frac{3}{2} \\
T=\frac{1}{2}
\end{array}\right)^{N}= \frac{-g^{2}}{8 q^{4}}\left(\begin{array}{r}
1 \\
-\frac{1}{2}
\end{array}\right)\left\{\left[(W+M)^{2}-\mu^{2}\right](W-M)\right. \\
& \times\left[2-a \ln \frac{a+1}{a-1}\right]+\left[(W-M)^{2}-\mu^{2}\right](W+M) \\
&\left.\times\left[-3 a+\left(\frac{3 a^{2}-1}{2}\right) \ln \frac{a+1}{a-1}\right]\right\} .
\end{aligned}
$$

The discontinuities of (6.10) are contained in the 
logarithm, which can be written in the form

$$
\ln \frac{a+1}{a-1}=\ln \left[\frac{W^{2}\left(W^{2}-M^{2}-2 \mu^{2}\right)}{W^{2} M^{2}-\left(M^{2}-\mu^{2}\right)^{2}}\right] .
$$

There is a short branch cut from $W=\left(M^{2}-\mu^{2}\right) / M$ to $\left(M^{2}+2 \mu^{2}\right)^{\frac{1}{2}}$ with the corresponding reflection on the negative real axis. There is also a branch cut from $-i \infty$ to $i \infty$. The short branch cut on the right corresponds to the pole in the static theory. Its reflection corresponds to a " $D$-wave pole" and has a relative strength $<\mu^{2} / M^{2}$. The branch cut on the imaginary axis, which is a distance of order $M$ from threshold in the $W$ plane, was pushed out to $\infty$ in the static theory.

We now proceed to approximate the discontinuities by a set of poles as motivated in the preceding section. From Eqs. (6.10) and (6.11) one finds that

$$
\begin{aligned}
\operatorname{Abs} g_{1+}(W)^{N} & =( \pm)\left(\begin{array}{r}
1 \\
-\frac{1}{2}
\end{array}\right) \frac{\pi g^{2}}{8 q^{4}}\left\{\left[(W+M)^{2}-\mu^{2}\right]\right. \\
& \times(W-M) a-\left[(W-M)^{2}-\mu^{2}\right] \\
& \left.\times(W+M) \frac{1}{2}\left(3 a^{2}-1\right)\right\} .
\end{aligned}
$$

This function is plotted in Fig. 9.

The sign in (6.12) is to be taken from Fig. 6. The short cut on the right extends over distances small compared to the distance from the cut to threshold. We shall therefore neglect the variation of the denominator in a Cauchy integral around this cut and integrate directly over the absorptive part. Doing this, one gets a pole whose residue is just that of the static theory:

$$
\left(\begin{array}{c}
1 \\
-\frac{1}{2}
\end{array}\right) \frac{g^{2}}{3(W-M)}
$$

Using the same procedure for the reflected cut, one finds that the residue of the pole is down by a factor $<\mu^{2} / M^{2}$ as previously indicated. Since we are concerned with calculating the $P$ waves, and since in addition to the fact that the residue is so small, the pole is a distance $2 M$ away from the region of interest, we shall neglect it.

In our first attempt to represent the cut along the imaginary axis we used a pair of conjugate poles, but in order to satisfy all the criteria of Sec. V we were ultimately forced to employ two pairs of conjugate poles. When combined with (6.13), these poles yield

$$
\begin{array}{r}
g_{1+}{ }^{N=} \frac{g^{2}}{3}\left(\begin{array}{r}
1 \\
-\frac{1}{2}
\end{array}\right)\left[\frac{1}{W-M}+\left(\frac{a}{W-W_{a}}+\frac{a^{*}}{W-W_{a}^{*}}\right)\right. \\
\left.+\left(\frac{b}{W-W_{b}}+\frac{b^{*}}{W-W_{b}^{*}}\right)\right],
\end{array}
$$

where

$$
\begin{array}{ll}
a=2.03, & W_{a}=2.12 M i, \\
b=-2.85 i, & W_{b}=4.32 M i .
\end{array}
$$

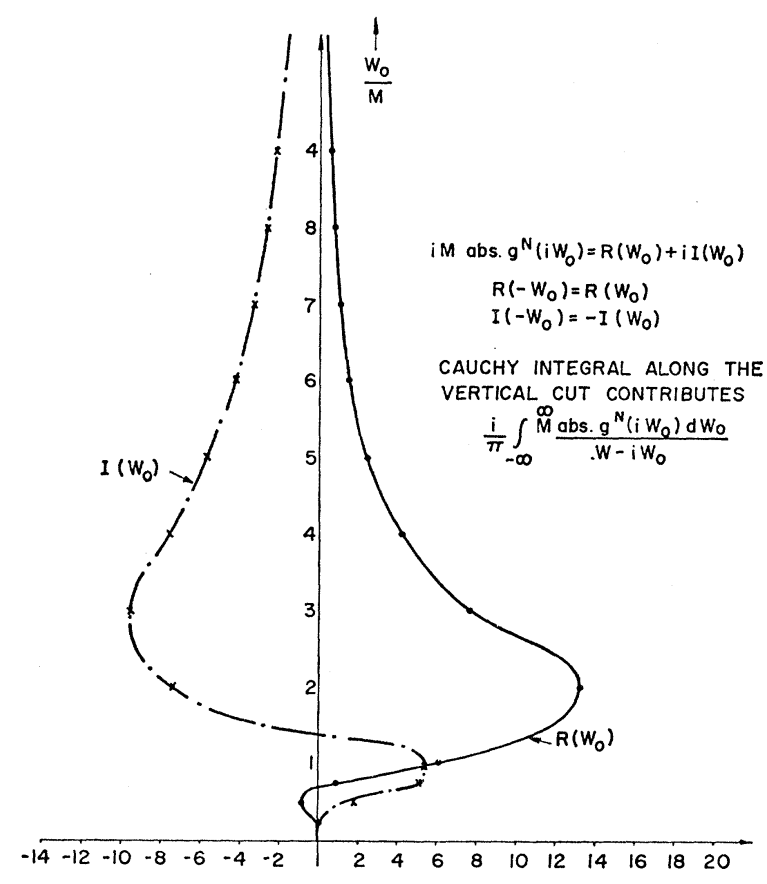

FIG. 9. Absorptive part of the single-nucleon term along the imaginary axis.

These poles reproduce the contribution of the Cauchy integral around the single-nucleon cut to within a few percent over the range of $W$ on the real axis, $6 M \gtrsim W$ $>M+\mu$ and $-M-\mu>W \gtrsim-6 M$. This is more than adequate for the integrals which we will have to take over this region.

Near the threshold $W=M+\mu, g_{1}$ is dominated by the familiar static pole at $W=M$. As $W$ increases, the poles on the imaginary axis take over and dominate the asymptotic behavior of Eq. (6.14), ${ }^{16}$

$$
\begin{gathered}
g_{1+}{ }^{N} \sim \frac{2 g^{2}}{W^{2}}\left(\begin{array}{r}
1 \\
-\frac{1}{2}
\end{array}\right) . \\
g_{1+} N \sim \frac{1.68 g^{2}}{W}\left(\begin{array}{r}
1 \\
-\frac{1}{2}
\end{array}\right) .
\end{gathered}
$$

This asymptotic behavior may be compared with the limit $\left(\left|e^{i \delta} \sin \delta\right| \leq 1\right)$ imposed by unitarity for real $W \rightarrow \infty$ :

$$
g_{1+}=\frac{W^{2} e^{i \delta_{1}+} \sin \delta_{1+}}{q^{3}} \leqslant \frac{8}{W} \sim \frac{g^{2}}{2 W} .
$$

It is clear that the unitarity condition must act so as to reduce the single-nucleon term for large $W$.

For purposes of orientation it is helpful to think of the pole at $W=M$ as producing a "long-range potential," which is especially important at low energies. The cut along the imaginary axis is then analogous to a "short-range potential," which influences high-energy

${ }^{16}$ The exact $g_{1+}{ }^{N}[\mathrm{Eq} .(6.10)]$ behaves asymptotically as 


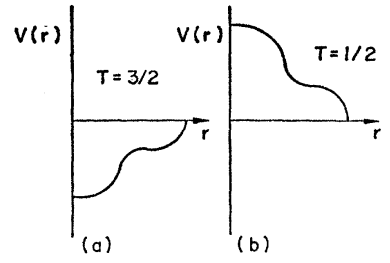

FIg. 10. Equivalent potentials in the $J=\frac{3}{2}, P$ states.

scattering strongly. The long- and short-range potentials are each attractive in the $T=\frac{3}{2}$ state, and repulsive in the $T=\frac{1}{2}$ state (Fig. 10). We shall find this potential analogy extremely helpful in interpreting our final results.

We now proceed to solve the problem that we have set up. To do this we use the $N / D$ method of Chew and Mandelstam. ${ }^{6}$ We let

$$
g_{1+}=N / D,
$$

where $N$ contains all the singularities on the left. That is, we write

$$
\begin{aligned}
N(W)=\frac{g^{2}}{3}\left(\begin{array}{r}
1 \\
-\frac{1}{2}
\end{array}\right)\left[\frac{D(M)}{W-M}+\left(\frac{a D\left(W_{a}\right)}{W-W_{a}}+\frac{a^{*} D^{*}\left(W_{a}\right)}{W-W_{a}^{*}}\right)\right. \\
\left.\quad+\left(\frac{b D\left(W_{b}\right)}{W-W_{b}}+\frac{b^{*} D^{*}\left(W_{b}\right)}{W-W_{b} *}\right)\right] \\
\equiv \sum_{i} \frac{R_{i} D\left(W_{i}\right)}{W-W_{i}} .
\end{aligned}
$$

We have chosen $N$ in such a way that

$$
N^{*}\left(W^{*}\right)=N(W) \text {. }
$$

We put the physical branch cut into $D$. Equation (6.19) and the property $g^{*}\left(W^{*}\right)=g(W)$ [Eq. (5.1)] imply

$$
D^{*}\left(W^{*}\right)=D(W)
$$

The normalization of $D$ is arbitrary up to this point. We choose to fix the normalization by setting

$$
D(W=M)=1,
$$

which enables us to compare readily with the Chew-Low effective-range formula. These properties of $D$ enable us to write

$$
\begin{array}{r}
D(W)=1+\frac{W-M}{\pi}\left[\int_{M+\mu}^{\infty} \frac{\operatorname{Im} D\left(W^{\prime}\right) d W^{\prime}}{\left(W^{\prime}-W\right)\left(W^{\prime}-M\right)}\right. \\
\left.+\int_{-\infty}^{-(M+\mu)} \frac{\operatorname{Im} D\left(W^{\prime}\right) d W^{\prime}}{\left(W^{\prime}-W\right)\left(W^{\prime}-M\right)}\right] .
\end{array}
$$

Since $N$ is real on the real axis, we can set

$$
\operatorname{Im} D=N \operatorname{Im} 1 / g
$$

in $(6.22) .{ }^{17} \mathrm{We}$ assume elastic scattering so that

$$
\operatorname{Im} 1 / g=-q^{3} / W^{2} \text {. }
$$

Substituting (6.24), (6.23), and (6.18) into (6.22), we have

$$
\begin{aligned}
D(W)=1- & \frac{W-M}{\pi} \sum_{i} R_{i} D\left(W_{i}\right) \int_{M+\mu}^{\infty} \frac{d W^{\prime} q^{3}\left(W^{\prime}\right)}{W^{\prime 2}} \\
\times & {\left[\frac{1}{\left(W^{\prime}-W\right)\left(W^{\prime}-M\right)\left(W^{\prime}-W_{i}\right)}\right.} \\
& \left.-\frac{1}{\left(W^{\prime}+W\right)\left(W^{\prime}+M\right)\left(W^{\prime}+W_{i}\right)}\right] .
\end{aligned}
$$

Evaluation of $D$ at the $W_{i}$ gives a set of linear equations which completely determine the $D\left(W_{i}\right)$, and the "effective" residues which appear in $N$.

The results are

$$
\begin{aligned}
T=\frac{3}{2} \quad D(2.12 M i) & =4.64-4.27 i, \\
D(4.32 M i) & =4.52-5.48 i, \\
D^{*}\left(W^{*}\right) & =D(W), \\
D=\frac{1}{2} \quad & D(2.12 M i)=0.61+0.11 i, \\
D(4.32 M i) & =0.33+0.14 i .
\end{aligned}
$$

The striking difference in the isotopic spin states can be interpreted in terms of the potential analogy (Fig. 10). The attractive short-range $T=\frac{3}{2}$ "potential" pulls in the wave function, greatly enhancing the effect of the potential on the scattering amplitude. According to our results this short-range potential essentially determines the $3-3$ resonance energy, thus playing a role analogous to the high-energy cutoff which determines the resonance energy in the static theory. On the other hand, the repulsive short-range $T=\frac{1}{2}$ potential pushes out the wave function so that the scattering amplitude becomes much less dependent on the strength of the repulsion. We recall that the determining characteristic of a hard core is its size and not its strength.

It follows from these considerations that the $T=\frac{3}{2}$ amplitude will be very sensitive to the details of the short-range interaction. In the next section, where we consider the crossed $\pi-N$ and $\pi-\pi$ channels, we shall find that we can treat long-range contributions from these channels accurately, but not the short-range contributions. Therefore our approach cannot be expected to give results of high quantitative accuracy in the $T=\frac{3}{2}$ state although we may hope to get the qualitative features correctly. For the $T=\frac{1}{2}$ state, however, the amplitude is much less sensitive to the de-

${ }^{17}$ If a different amplitude were used, the change in asymptotic behavior would be the same for both $g$ and $N$. Therefore theasymptotic behavior of $\operatorname{Im} D$ would be unchanged and the con-
vergence of Eq. (6.22) would be unaffected. 


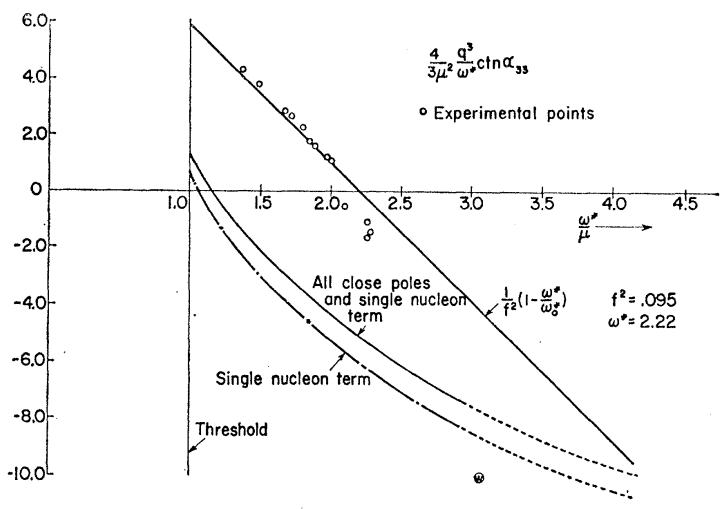

Fig. 11. Chew-Low effective range plot for the $J=\frac{3}{2}, T=\frac{3}{2} P$ state.

tails of the short-range interaction, and we would hope that here we could arrive at more quantitative results. ${ }^{18}$

To compare our results with the experimental effective range plots we calculate the function

$$
\begin{aligned}
& \frac{4}{3 \mu^{2}} \frac{q^{3}}{W-M} \operatorname{ctn} \delta_{1+} \\
& =\frac{4}{3 \mu^{2}} \frac{W^{2}}{W-M} \operatorname{Re}^{\frac{1}{g_{1+}}}=\frac{4}{3 \mu^{2}} \frac{W^{2}}{(W-M) \sum_{i} \frac{R_{i} D\left(W_{i}\right)}{W-W_{i}}} \\
& \quad \times\left\{1-\frac{(W-M)}{\pi} \sum_{i} R_{i} D\left(W_{i}\right) P \int \frac{q^{\prime 3} d W^{\prime}}{W^{\prime 2}}\right. \\
& \quad \times\left[\frac{1}{\left(W^{\prime}-W\right)\left(W^{\prime}-M\right)\left(W^{\prime}-W_{i}\right)}\right. \\
& \left.\left.-\frac{1}{\left(W^{\prime}+W\right)\left(W^{\prime}+M\right)\left(W^{\prime}+W_{i}\right)}\right]\right\} .
\end{aligned}
$$

This function is plotted in Fig. 11 for the $T=\frac{3}{2}$ state. We have also calculated the phase shifts themselves in the $T=\frac{3}{2}$ and the $T=\frac{1}{2}$ states, and they are plotted in Figs. 12 and 13. We shall discuss these in the next section, after the long-range contributions from the crossed $\pi-N$ and $\pi-\pi$ channels have been added.

The error which our replacement of branch cuts by poles introduces can be estimated by substituting our calculated $D$ into the exact integral equation for $D$. Estimates of this type indicate that our $T=\frac{3}{2}, P$-wave solution contains errors of order $20 \%$ or less, and the $T=\frac{1}{2}$ solution is good to a few percent in the low-energy region. ${ }^{19}$

We recall that in choosing our amplitudes, we had failed to guarantee the correct threshold behavior of

\footnotetext{
${ }^{18}$ It appears that this is also the situation in the $J=\frac{1}{2}$ state, and work is now in progress on this state.

${ }^{19}$ We have also checked that no ghosts $(D=0)$ appear anywhere near the physical region in our solution.
}

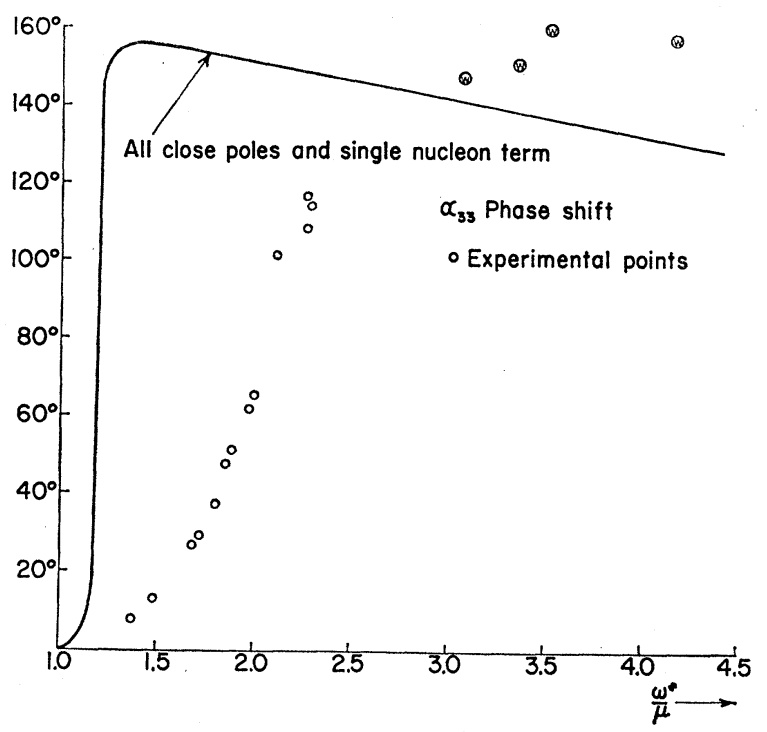

FIG. 12. Phase shift in the $J=\frac{3}{2}, T=\frac{3}{2} P$ state.

the $D$ waves. We are now in a position to check on what the resulting $D$-wave behavior is. Using

$$
g_{2-}(W)=-g_{1+}(-W),
$$

we find that the $D$ waves are negative and small in the region $M+\mu<W<2 M$. Since we have not treated the $D$ threshold correctly and have neglected singularities lying near the $D$ cut, we have no reason to believe these results. We wish to point out only that the inadequacy of our treatment of the low-energy $D$ state does not appear to introduce a large error in our calculation of the $P$ states.

\section{TREATMENT OF THE CROSSED STATES}

In this section we want to improve the treatment described in the previous section by including the information that we have on the crossed states. We discuss first the contributions of the crossed $\pi-N$ cut.

\section{A. Crossed $\pi-N$ Cut}

As has been previously indicated, that part of the crossed $\pi-N$ cut lying on the real axis can be related by crossing symmetry to physical $\pi-N$ scattering. We

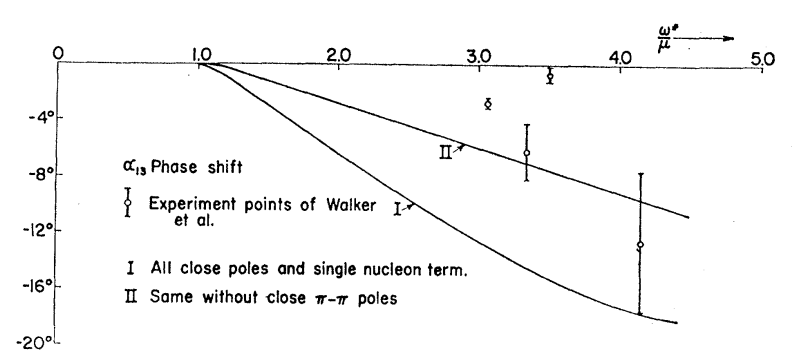

FIG. 13. Phase shift in the $J=\frac{3}{2}, T=\frac{1}{2} P$ state. 
shall now use the experimental information that $\pi-N$ scattering is dominated by the $3-3$ resonance at low energies and shall investigate the consequences of keeping only $\operatorname{Im} f_{1+}{ }^{3}$ in $\operatorname{Im} f$ [Eqs. (2.17), (2.18)]:

$$
\begin{aligned}
& \operatorname{Im} f_{1}(s, u)=3 x \operatorname{Im} f_{1+}(s), \\
& \operatorname{Im} f_{2}(s, u)=-\operatorname{Im} f_{1+}(s) .
\end{aligned}
$$

This gives us

$$
\begin{array}{r}
\operatorname{Im} A^{ \pm}(s, u)=8 \pi W\left[\frac{W+M}{(W+M)^{2}-\mu^{2}} 3 x\right. \\
\left.\quad+\frac{W-M}{(W-M)^{2}-\mu^{2}}\right] \operatorname{Im} f_{1+} \pm(s), \\
\operatorname{Im} B^{ \pm}(s, u)=8 \pi W\left[\frac{1}{(W+M)^{2}-\mu^{2}} 3 x\right. \\
\left.\quad-\frac{1}{(W-M)^{2}-\mu^{2}}\right] \operatorname{Im} f_{1+}{ }^{ \pm}(s) .
\end{array}
$$

We can calculate the contribution of the crossed $\pi-N$ cut from these formulas by using crossing symmetry:

$$
\begin{aligned}
& \operatorname{Im} A^{ \pm}(s, u)= \pm \operatorname{Im} A^{ \pm}(u, s), \\
& \operatorname{Im} B^{ \pm}(s, u)= \pm \operatorname{Im} B^{ \pm}(u, s) .
\end{aligned}
$$

If we now substitute a sharp resonance approximation, ${ }^{2}$

$$
\begin{aligned}
\operatorname{Im} f_{1+}{ }^{3}(s) & =\left(8 \pi W_{R} / 3\right)(f / \mu)^{2} q^{2} \delta\left(W^{2}-W_{R}{ }^{2}\right) \\
f^{2} & =(\mu / 2 M)^{2} g^{2}
\end{aligned}
$$

and use

$$
f_{1+}^{ \pm=\frac{1}{3}}\left(\begin{array}{r}
2 \\
-1
\end{array}\right) f_{1+}^{3}
$$

then we find

$$
\begin{aligned}
\operatorname{Im} A(u, s) & \left(\begin{array}{c}
T=\frac{3}{2} \\
T=\frac{1}{2}
\end{array}\right)=\left(\begin{array}{l}
1 \\
4
\end{array}\right) \frac{64 \pi^{2} W_{R^{2}}^{2}}{9} \delta\left(u-W_{R}^{2}\right)\left(\frac{f}{\mu}\right)^{2} q_{r}^{2} \\
\times & {\left[\frac{W_{R}+M}{\left(W_{R}+M\right)^{2}-\mu^{2}} 3 x^{\times}+\frac{W_{R}-M}{\left(W_{R}-M\right)^{2}-\mu^{2}}\right], } \\
\operatorname{Im} B(u, s) & \left(\begin{array}{l}
T=\frac{3}{2} \\
T=\frac{1}{2}
\end{array}\right)=-\left(\begin{array}{l}
1 \\
4
\end{array}\right) \frac{64 \pi^{2} W_{R}^{2}}{9} \delta\left(u-W_{R}^{2}\right)\left(\frac{f}{\mu}\right)^{2} q_{r}^{2} \\
& \times\left[\frac{3 x^{\times}}{\left(W_{R}+M\right)^{2}-\mu^{2}}-\frac{1}{\left(W_{R}-M\right)^{2}-\mu^{2}}\right]
\end{aligned}
$$

where by Eq. (4.14)

$$
x^{\times}=1+\frac{2 W_{R^{2}}\left(2 M^{2}+2 \mu^{2}-s-W_{R}^{2}\right)}{W_{R}^{4}-2 W_{R}^{2}\left(M^{2}+\mu^{2}\right)+\left(M^{2}-\mu^{2}\right)^{2}} .
$$

In our procedure of approximating the dynamic singularities by poles we also need the Cauchy integral around the cuts on the left. Instead of directly calculat- ing the Cauchy integral from the absorptive part, we shall make use of a procedure which in practice makes approximating the cuts by poles easier. We construct the functions

$$
\begin{aligned}
& A(s, x) \times=\frac{1}{\pi} \int_{(M+\mu)^{2}}^{\infty} \frac{\operatorname{Im} A\left(u^{\prime}, s\right) d u^{\prime}}{u^{\prime}-u} \\
& B(s, x)^{\times}=\frac{1}{\pi} \int_{(M+\mu)^{2}}^{\infty} \frac{\operatorname{Im} B\left(u^{\prime}, s\right) d u^{\prime}}{u^{\prime}-u} .
\end{aligned}
$$

These functions have a branch cut along the crossed $\pi-N$ cut and are analy tic everywhere else in the $s$ plane. They also have the correct discontinuities across the cut. We find by substituting from Eqs. (7.7), (7.8) and projecting out partial waves that

$$
\begin{array}{r}
A_{l}(s) \times=\frac{64 \pi W_{R}^{2}}{9}\left(\begin{array}{l}
1 \\
4
\end{array}\right)\left(\frac{f}{\mu}\right)^{2} q_{r}{ }^{2}\left[\frac{W_{R}+M}{\left(W_{R}+M\right)^{2}-\mu^{2}} 3 x^{\times}\right. \\
\left.+\frac{W_{R}-M}{\left(W_{R}-M\right)^{2}-\mu^{2}}\right] \int_{-1}^{1} \frac{P_{l}(x) d x}{u_{R}-u(s, x)}, \\
B_{l}(s)^{\times}=\frac{-64 \pi W_{R^{2}}}{9}\left(\begin{array}{l}
1 \\
4
\end{array}\right)\left(\frac{f}{\mu}\right)^{2} q_{r}{ }^{2}\left[\frac{3 x^{\times}}{\left(W_{R}+M\right)^{2}-\mu^{2}}\right. \\
\left.-\frac{1}{\left(W_{R}-M\right)^{2}-\mu^{2}}\right] \int_{-1}^{1} \frac{P_{l}(x) d x}{u_{R}-u(s, x)}
\end{array}
$$

The equation for the contribution of these functions to our amplitude $g_{1+}$ then becomes

$$
\begin{aligned}
g_{1} \times=\frac{W_{R}^{2}}{18 q^{4}} & \left(\begin{array}{l}
1 \\
4
\end{array}\right)\left(\frac{f}{\mu}\right)^{2} q_{r^{2}}\left\{\left[(W+M)^{2}-\mu^{2}\right]\right. \\
& \times\left[\frac{3 x^{\times}\left(W_{R}+2 M-W\right)}{\left(W_{R}+M\right)^{2}-\mu^{2}}+\frac{W_{R}-2 M+W}{\left(W_{R}-M\right)^{2}-\mu^{2}}\right] \\
& \times\left[2-a \ln \frac{a+1}{a-1} \mid+\left[(W-M)^{2}-\mu^{2}\right]\right. \\
& \times\left\lfloor\frac{3 x^{\times}\left(W_{R}+2 M+W\right)}{\left(W_{R}+M\right)^{2}-\mu^{2}}+\frac{W_{R}-2 M-W}{\left(W_{R}-M\right)^{2}-\mu^{2}}\right] \\
& \left.\times\left[-3 a+\left(\frac{3 a^{2}-1}{2}\right) \ln \frac{a+1}{a-1}\right]\right\},
\end{aligned}
$$

with

$$
a=\frac{s+W_{R^{2}}-2 M^{2}-2 \mu^{2}}{2 q^{2}}-1 \text {. }
$$

When we take the absorptive part of this expression, we find that the cuts run the length of the imaginary axis and along a short interval on the real axis,

$$
0.59 M \leq|W| \leq 0.76 M \text {. }
$$


The contribution from the short cuts can be approximated by a pole of the form ${ }^{20}$

$$
g_{1+} \times=\frac{g^{2}}{54 \pi}\left(\frac{1}{W-0.68 M}\right)\left(\begin{array}{l}
1 \\
4
\end{array}\right) .
$$

In terms of the potential analogy, this expression gives the long-range part of the force coming from channel II. When we next try to evaluate the shortrange contribution of the force coming from this crossed process, we find that the absorptive part along the vertical axis contains angles $x^{\times}$that lie in a region such that the Legendre polynomial expansion in channel II diverges. Formally, the extent of the region of convergence of the Legendre expansion can be determined from the regions in which the two-dimensional spectral functions $\rho_{i j}$ fail to vanish. This analysis is carried out in Appendix C. The limitation on the use of the Legendre expansion can in principle be overcome by direct analysis of the spectral functions. This procedure has so far appeared to be prohibitively difficult to carry out. We have seen that our analysis of the $T=\frac{3}{2}$ state depended on the details of the short-range force and therefore a reliable treatment of these shortrange forces must be developed before a quantitatively satisfactory calculation can be made in this state. ${ }^{21}$ However, we have also seen that in the $T=\frac{1}{2}$ state (and also, it appears, in the $J=\frac{1}{2}$ states) the amplitude is insensitive to the details of the short-range force coming from the crossed processes unless it should turn out that these are so strongly attractive as to dominate the repulsive short-range contributions from the single-nucleon term.

Keeping the preceding considerations in mind we shall only include the known close-lying singularities coming from channel II and take

$$
g_{1+}(W)^{\times}=\frac{g^{2}}{54 \pi}\left(\begin{array}{l}
1 \\
4
\end{array}\right) \frac{1}{W-0.68 M} .
$$

$$
\text { B. } \pi-\pi \mathrm{Cut}
$$

For the amplitude corresponding to channel III we shall use the work of Frazer and Fulco who have proposed that a $T=1, J=1, \pi-\pi$ resonance will improve the agreement between theory and experiment for the electromagnetic structure of the nucleon. ${ }^{8}$ The interaction in any other state in channel III could also, of course, be included if it were known. We shall be primarily interested here, however, in pursuing the consequences of the proposed $\pi-\pi$ resonance.

\footnotetext{
${ }^{20}$ The residue in Eq. (7.15) is also equal to

$$
1 / \pi \int_{0.59 M}^{0.76 M} \operatorname{Im} g_{1+}(W) d W \text {. }
$$

${ }^{21} \mathrm{We}$ attempted to cut off the diverging polynomial contributions but found in all cases that our results were extremely cutoffdependent.
}

We first note that if we have only $T=1$, then

$$
\begin{aligned}
& A_{\pi \pi^{+}}=B_{\pi \pi^{+}}=0, \\
& A_{\pi \pi^{-}}=\frac{1}{2} A_{\pi \pi^{1}}, \\
& B_{\pi \pi^{-}}=\frac{1}{2} B_{\pi \pi^{1}} .
\end{aligned}
$$

We keep only the $J=1$ state in Eqs. (2.37), (2.38) and obtain

$$
\begin{aligned}
& B_{\pi \pi^{-}}=6 \pi \sqrt{2} f_{1-}{ }^{-}(t), \\
& A_{\pi \pi^{-}}=-12 \pi(\zeta / p)\left[f_{1+}{ }^{-}-(M / \sqrt{2}) f_{1-}{ }^{-}\right] y,
\end{aligned}
$$

where

$$
y=\left(s+\frac{1}{2} l-M^{2}-\mu^{2}\right) / 2 p \zeta,
$$

and the variables refer to channel III. Frazer and Fulco do not directly give the helicity amplitudes $f_{1_{ \pm}}$but give quantities $\Gamma_{i}$ related to the $f$ 's by ${ }^{8,22}$

$$
\begin{aligned}
& \Gamma_{1}(t)=-\frac{M}{p^{2}}\left[\frac{E^{2}}{\sqrt{2} M} f_{1-}-(t)-f_{1+}-(t)\right], \\
& \Gamma_{2}(t)=\frac{-1}{2 p^{2}}\left[f_{1+}-(t)-\frac{M}{\sqrt{2}} f_{1-}-(t)\right] .
\end{aligned}
$$

The quantities $\Gamma_{i}(t)$ within the framework of their calculation are directly related to the electromagnetic form factor of the pion (we are actually only interested in imaginary parts),

$$
\operatorname{Im} \Gamma_{i}(t)=-\left(2 E / q^{3}\right)\left[g_{i}^{v}(t)_{0} / e\right] \operatorname{Im} F_{\pi}(t),
$$

where $g_{i} v(t)_{0}$ is the spectral function of the appropriate electromagnetic form factor of the nucleon calculated in the absence of $\pi-\pi$ interaction. They further give

$$
F_{\pi}(t)=\frac{11.3}{11.5-t / \mu^{2}-2.32 i} .
$$

and from Chew et al..$^{23}$ we have

$$
\begin{aligned}
& g_{2} v\left(11.5 \mu^{2}\right)_{0}=0.61 e f^{2} / \mu, \\
& g_{1}{ }^{v}\left(11.5 \mu^{2}\right)_{0}=1.85 e f^{2} .
\end{aligned}
$$

To simplify our calculations we shall make the approximation of a sharp resonance and write

$$
\begin{aligned}
& \operatorname{Im} \Gamma_{1}=\gamma_{1} \delta\left(t-t_{R}\right), \\
& \operatorname{Im} \Gamma_{2}=\left(\gamma_{2} / M\right) \delta\left(t-t_{R}\right),
\end{aligned}
$$

where we take $t_{R}=11.5 \mu^{2}$ from Eq. (7.23) and determine $\gamma$ by integrating over both sides of Eq. (7.22). Using

$$
\int_{-\infty}^{\infty} \operatorname{Im} F_{\pi}(t) d t=35.5 \mu^{2},
$$

${ }^{22} f_{1+}-$ means $J=1$ and helicity + and isotopic spin amplitude ${ }^{23}$ G. F. Chew, R. Karplus, S. Gasiorowicz, and F. Zachariasen, Phys. Rev. 110, 265 (1958). 
we find that

$$
\begin{aligned}
& \gamma_{1}=-6.9, \\
& \gamma_{2}=-15.6 ;
\end{aligned}
$$

and from Eqs. (7.17), (7.18),

$$
\begin{aligned}
& \operatorname{Im} B_{\pi \pi^{-}}=-12 \pi\left(\gamma_{1}+2 \gamma_{2}\right) \delta\left(t-t_{R}\right), \\
& \operatorname{Im} A_{\pi \pi^{-}}=12 \pi\left(\gamma_{2} / M\right)\left(s+t_{R} / 2-M^{2}-\mu^{2}\right) \delta\left(t-t_{R}\right) .
\end{aligned}
$$

We use these last two equations as the input information for channel III.

If, as in the last section, we calculate the functions

$$
\begin{aligned}
& A^{-}(s, x)^{\pi \pi}=\frac{1}{\pi} \int_{4 \mu^{2}}^{\infty} \frac{\operatorname{Im} A_{\pi \pi}-\left(t^{\prime}, s\right) d t^{\prime}}{t^{\prime}-t}, \\
& B^{-}(s, x)^{\pi \pi}=\frac{1}{\pi} \int_{4 \mu^{2}}^{\infty} \frac{\operatorname{Im} B_{\pi \pi}-\left(t^{\prime}, s\right) d t^{\prime}}{t^{\prime}-t},
\end{aligned}
$$

then these functions have a branch cut along the $\pi-\pi$ cut and are analytic everywhere in the $s$ plane. They also have the correct discontinuities across the cut. By projecting the appropriate partial waves out of this expression and combining the results we find

$$
\begin{aligned}
& g_{1+}(s)^{\pi \pi}\left(\begin{array}{l}
T=\frac{3}{2} \\
T=\frac{1}{2}
\end{array}\right) \\
&=\left(\begin{array}{r}
-1 \\
2
\end{array}\right) \frac{1}{32 \pi q^{2}}\left\{\left[(W+M)^{2}-\mu^{2}\right] \frac{6}{q^{2}}\right. \\
& \times\left(-2-A \ln \frac{A-1}{A+1}\right)\left[\frac{\gamma_{2}}{M}\left(s+\frac{t_{R}}{2}-M^{2}-\mu^{2}\right)\right. \\
&\left.-(W-M)\left(\gamma_{1}+2 \gamma_{2}\right)\right]+\left[(W-M)^{2}-\mu^{2}\right] \frac{3}{q^{2}} \\
& \times\left[-6 A+\left(1-3 A^{2}\right) \ln \frac{A-1}{A+1}\right] \\
& \times\left[-\frac{\gamma_{2}}{M}\left(s+\frac{t_{R}}{2}-M^{2}-\mu^{2}\right)\right. \\
&\left.\left.-(W+M)\left(\gamma_{1}+2 \gamma_{2}\right)\right]\right\}
\end{aligned}
$$

with

$$
A=1+t_{R} / 2 q^{2} \text {. }
$$

This function has a cut on the circle $W=\left(M^{2}-\mu^{2}\right)^{\frac{1}{2}} e^{i x}$ which starts at

$$
\begin{array}{r}
|\chi| \geqslant 11^{\circ} 45^{\prime}, \\
|\pi-\chi| \geqslant 11^{\circ} 45^{\prime},
\end{array}
$$

and continues to the vertical axis where it runs from $-\infty$ to $+\infty$. We are again here faced with the problem that the discontinuity along the cut will refer to angles $\phi$ in channel III for which the Legendre expansion diverges. The region of convergence of this expansion is discussed in Appendix $\mathrm{C}$ and is shown on Fig. 5. We are again only justified in using the contribution of the close-lying singularities in channel III. It happens that the contributions we are including from channel III separate conveniently into long-range and shortrange terms, because the angular dependence of the crossed $J=1$ state makes "intermediate-range" contributions from this particular state small. The contribution to the function $g_{1+}{ }^{(1,3)}(W)^{\pi \pi}$ from the close part of the circle is well-represented by

$$
\begin{aligned}
g_{1+}(W)^{\pi \pi} & =\left(\begin{array}{r}
-1 \\
2
\end{array}\right)(1.88 i) \\
& \times\left[1 /\left(W-M e^{i \chi_{0}}\right)-1 /\left(W-M e^{-i \chi_{0}}\right)\right],
\end{aligned}
$$

where $\chi_{0}=12^{\circ} 50^{\prime}$.

The neglect of the short-range interactions has the same status here as for the crossed $\pi-N$ contributions.

We are now in a position to add to our treatment of the singular-nucleon term the contributions of the close-lying singularities in the crossed channels as represented by Eqs. (7.16) and (7.36). We proceed in exactly the same fashion as indicated in Sec. VI. We find that

$$
\begin{aligned}
D^{3}\left(W_{R}\right) & =2.30, & D^{1}\left(W_{R}\right) & \cong 1, \\
D^{3}\left(M e^{i \chi_{0}}\right) & =1.30-1.07 i, & D^{1}\left(M e^{i \chi_{0}}\right) & =0.97+0.08 i, \\
D^{3}(2.12 M i) & =4.07-3.68 i, & D^{1}(2.12 M i) & =0.61+0.11 i, \\
D^{3}(4.32 M i) & =3.91-4.72 i, & D^{1}(4.32 M i) & =0.33+0.14 i .
\end{aligned}
$$

The results for the scattering amplitudes and phase shifts along the real axis are given in Figs. 11, 12, and 13. The experimental results as summarized by Puppi and Stanghellini, ${ }^{24}$ Ashkin et al..$^{25}$ and Walker et al. ${ }^{26}$ are also included in these figures. It is seen that the long-range $\pi-\pi$ interaction is responsible for $50 \%$ of the $\alpha_{13}$ phase shift in our calculation, while making only a very small contribution to $\alpha_{33}$. One reason for this difference is that the ratio of $\pi-\pi, T=1$ to singlenucleon contributions is four times greater in the $T=\frac{1}{2}$ than in the $T=\frac{3}{2}$ amplitude (for $J=\frac{3}{2}$ ) due to isotopic spin factors. The curve of $\alpha_{13}$ without the $\pi-\pi$ interaction is almost identical to the results of Chew, Goldberger, Low, and Nambu. ${ }^{2}$

Using exactly the same techniques as in Sec. VII A, we have also calculated the long-range contributions from the crossed $\pi-N, S$ and small $P$ states. These contributions are small and can be ignored. We estimate that a $\pi-\pi S$-state phase shift of order $30^{\circ}$ would give not more than one-third of the long-range contribution from the $\pi-\pi P$ state to the $T=\frac{3}{2}$ state (and one-sixth of the $\pi-\pi P$-state contribution to the $T=\frac{1}{2}$ state).

${ }^{24}$ G. Puppi and A. Stanghellini, Nuovo cimento 5, 1305 (1957).

${ }^{25} \mathrm{~J}$. Ashkin, J. P. Blaser, F. Feiner, and M. O. Stern, Phys. Rev. 105, 724 (1957).

${ }_{26}$ W. D. Walker, W. D. Shephard, and J. Davis, Phys. Rev. 118, $1612(1960)$. 
Thus there is some basis for ignoring this (unknown) $\pi-\pi S$-state contribution as well.

Although these results indicate that it is a good approximation to ignore long-range contributions from nonresonating, low-energy crossed reactions, the region of convergence of the Legendre expansion includes other crossed contributions which are harder to estimate. The crossed $\pi-N$ singularity on the real axis, at $-0.5 M \leqslant W \leqslant 0.5 M$, involves physical $\pi-N$ scattering at energies ranging from just above the $3-3$ resonance to $\infty$. This region does not appear to give a large contribution. ${ }^{27}$ As $W$ approaches the limit of convergence on the circular $\pi-\pi$ cut (Fig. 5), the singularities are related to $N+\bar{N} \rightarrow n \pi$ with energies up to $t \sim M^{2}$. Arguments, based either on phase space or putting pairs of pions into $T=1, J=1$ resonance states, indicate that the $4 \pi$ intermediate state should not be especially prominent for $t \leqslant M^{2}$. However, if a $3 \pi$ bound state, $X$, exists with $T=0$ and $J=1,{ }^{28}$ the $\pi X$ state would contribute well below $t=M^{2}$. Furthermore, unlike the singularities on the real axis, the processes $N+\bar{N} \rightarrow n \pi$ which contribute on the circular $\pi-\pi$ cut $\left(t<4 M^{2}\right)$ are nonphysical and cannot be bounded by unitarity (just as contributions with $t<4 M^{2}$ cannot be bounded in the nucleon electromagnetic structure problem).

To summarize, we believe we can reliably calculate the singularities nearest to the physical region, as well as the various single nucleon singularities. Proceeding away from the physical region, but still within the region where the Legendre expansion converges, we find singularities which can only be estimated at present. Beyone that, outside of the region of convergence, lie singularities which we cannot even estimate at this time. As in most applications of dispersion relations we have been obliged to go ahead and see what results we could obtain by ignoring those contributions which we could not calculate.

\section{SHORT-RANGE MODIFICATIONS AND INELASTIC SCATTERING}

In the previous sections we have obtained the $T=\frac{3}{2}$, $J=\frac{3}{2}$ resonance, but at too low an energy. The longrange interactions entering into our calculations are supposed to be quite accurate, so that short-range interactions we have not taken into account are presumably responsible for the differences between our results and experiment. It is of interest to consider the magnitude of the modification required in the shortrange interaction to bring the resonance energy into agreement with experiment, and also whether the energy dependence of the phase shift is given correctly

${ }^{27}$ If we assume a constant $\pi-N$ cross section in the high-energy limit, the integrated contribution to the discontinuity near $W=0$ behaves $\sim W^{2}$.

28 The possible existence of such a bound state has been proposed by G. F. Chew, Phys. Rev. Letters 4, 142 (1960). once the theory has been modified to give the correct resonance energy.

In order to discuss these questions we adopt the approximate procedure of multiplying all residues of $N$ on the imaginary axis $[\mathrm{Eq} .(6.18)]$ by a common factor z. We believe our treatment of the nearby singularities to be correct, and therefore leave these singularities unchanged. We thus modify the problem by introducing one parameter which represents an over-all reduction of the short-range interaction.

Carrying out the rest of the calculation as before, we find that $z=0.6$ leads to a resonance at the experimental energy. This suggests that the combined effect of short-range contributions from channels II and III must weaken the single-nucleon potential for $T=\frac{3}{2}$ by about $40 \%{ }^{29}$ As we have seen, a change of this type in the $T=\frac{1}{2}$ state would have very little effect on lowenergy scattering in that state.

When we proceed to study the energy dependence of the $T=\frac{3}{2}$ amplitude with $z=0.6$, we obtain the experimental effective-range curve below resonance, but above resonance our results curve upward instead of the downward trend indicated by experiment (Fig. 11). More complicated modifications of the discontinuities along the imaginary axis, involving more parameters than $z$, do not easily lead to improved agreement with experiment.

Inelastic scattering at high energies in channel I, which we have ignored previously, offers more promise in explaining the data above resonance. An accurate treatment of inelastic scattering is not possible at present, but estimates can be made which indicate the direction and approximate magnitude of the effects to be expected. If inelastic scattering is present, Eq. (6.24) is replaced by

$$
\begin{aligned}
\operatorname{Im} D & =N \operatorname{Im} 1 / g \\
& =-N\left(q^{3} / W^{2}\right)\left(\sigma_{T} / \sigma_{E}\right),
\end{aligned}
$$

where $\sigma_{T}$ and $\sigma_{E}$ are the total and elastic scattering cross sections for the particular state in question. ${ }^{30}$ Thus our former expression for $\operatorname{Im} D$ is multiplied by a factor which is always greater than or equal to one. It is equal to one for pure elastic scattering and it takes the value two when the partial wave is completely absorbed. If the real part of the phase shift is large, as in the 3-3 state where it decreases slowly through $90^{\circ}$ (Fig. 12) then the elastic scattering will dominate and we have $\sigma_{E} \sim \sigma_{T}$.

We have made an approximate calculation assuming that $\sigma_{T} \sim 1.3 \sigma_{E}$ above $600 \mathrm{Mev}$ in the laboratory. In this calculation the short-range interaction was reduced in such a way as to keep the resonance energy fixed at its experimental value. The results obtained from

${ }^{29}$ When the effects of inelastic scattering in channel I are included, one finds that this estimate of $40 \%$ has to be increased somewhat.

${ }^{30}$ We wish to thank P. Federbush for an informative discussion on this point. 

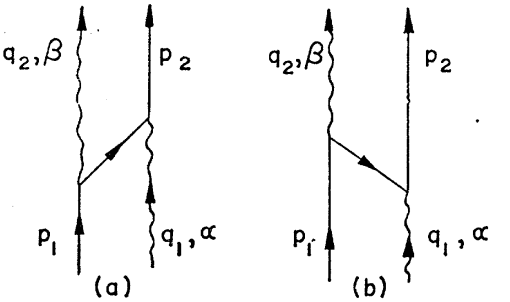

Fig. 14. Time-ordered diagrams for the single-nucleon term which contributes to the $J=\frac{3}{2}$ state.

this qualitatively reasonable treatment reproduce the effective-range formula below resonance, and turn below the effective-range curve just after resonance in approximate agreement with experiment. At higher energies the phase shift levels off at $135^{\circ}$ or $140^{\circ}$. Estimates made with larger inelasticity factors lead to somewhat higher maximum phase shifts.

The effect of inelastic scattering on the low-energy $T=\frac{1}{2}$ amplitude can also be estimated. If conditions are such that $\sigma_{E} \gtrsim \frac{1}{2} \sigma_{T}$ at all energies, the effect is small.

\section{CONCLUSION}

We are making use of partial wave dispersion relations derived from the double dispersion relations for $\pi-N$ scattering. The fully covariant character of these equations allows, in principle, cutoff-free calculations of all quantities except the physical mass and coupling constants. Thus there is some hope of obtaining the position of the $3-3$ resonance and the $S$-wave scattering lengths at threshold. In this paper we have applied the equations to the $J=\frac{3}{2}, P$ state.

We first took the "single-nucleon" diagram (Fig. 4) into account. To display the physical nature of the interaction arising from this diagram we can split it into its two time orderings (Fig. 14). Figure 14 (a) may may be described as the exchange of a nucleon, and it can be shown that it contributes both a "long-range" and a "short-range" interaction. Figure $14(\mathrm{~b})$ represents the creation and annihilation of a pair, and is all "short-range." The "short-range" contributions from the single-nucleon term are a feature of our calculation which was not present in the static theory at finite energies because there the nucleon mass was taken to be infinite. We have also taken into account "long-range" contributions from $\pi-N$ and $\pi-\pi$ scattering in crossed channels.

For practical reasons we have not taken into account inelastic scattering, and many "shorter-range" contributions. The neglect of inelastic scattering means of course that our calculations are not valid at energies where inelastic scattering is large. ${ }^{31}$ If the short-range, single-nucleon interaction is strongly repulsive, as in the $T=\frac{1}{2}$ state, we hope that we may have a quantita-

31 There is also some question as to the meaning of the small phase shifts at low energy because of charge-dependent effects. These effects have been considered by D. M. Greenberger, Phys. Rev. 117, 1387 (1960), who shows that they decrease with increasing energy and are unimportant at energies above resonance. tively reliable treatment at low energies. This treatment will not be changed by taking more short-range effects into account unless they are strongly attractive. We do have to assume that the $\pi-\pi$ interaction is given and "intermediate-range" multiple pion contributions are not too important. In the attractive $T=\frac{3}{2}$ state, on the other hand, our treatment can only be qualitative because the resonance energy is sensitive to small modifications of the short-range interaction. Therefore our methods do not yet suffice to determine the resonance energy accurately although they do predict a resonance structure for the $3-3$ phase shift.

Our results for the $J=\frac{3}{2}, P$ states are shown in Figs. 11,12 , and 13 . It can be seen that the $\pi-\pi$ resonance which has been proposed ${ }^{8}$ for the $T=1, J=1$ two-pion state changes the $T=\frac{1}{2}$ state by $50 \%$ and the $T=\frac{3}{2}$ state by very little. ${ }^{32}$

At present we are applying the same methods to the $J=\frac{1}{2}$ states. Here the short-range single-nucleon interaction is again repulsive so that we may be able to obtain quantitatively reliable results.

\section{ACKNOWLEDGMENTS}

We are indebted to G. F. Chew and S. Mandelstam for constant advice and encouragement. Some correspondence with W. Frazer was also helpful.

\section{APPENDIX A: BEHAVIOR OF AMPLITUDE AT $W=0$}

In this appendix we reproduce an argument of Mandelstam, ${ }^{12}$ to the effect that the partial wave amplitude

$$
h_{l}=e^{i \delta} \sin \delta / q^{2 l+1}
$$

is in general nonzero at $s=0$ when a finite number of coupled partial waves is considered. [It is not known whether $h_{l}(W=0)$ vanishes as the number of coupled partial waves approaches $\infty$.] The factor $q^{-2 l}$ would appear to introduce a zero because $q^{-2} \sim s$ near $s=0$ [Eq. (2.9)]. We shall show that this factor is actually cancelled by another factor, $s^{-l}$, coming from the partial wave projection so that there is no reason to believe that $h_{l}=0$ at $W=0$. We present this argument with the hope of stimulating the development of a more complete understanding of this subject in the future.

Since the possibility of a zero due to $q^{-2 l}$ arises whether or not the particles have spin, consider the case with no spin. In this case there are no kinematic singularities and no reflection principle, so (A.1) is appropriate rather than more complicated functions such as Eq. (3.2). We further restrict our attention to $S$ and $P$ waves; it will be clear how to extend the argument to progressively higher $l$. The total amplitude

${ }^{32}$ Recently J. Bowcock, W. Cottingham, and D. Lurié, preprint (to be published) have added the Frazer-Fulco $\pi-\pi$ resonance to the fixed momentum transfer dispersion relations of reference 2 . At low energies they obtain $\pi-\pi$ effects for the $P$ states which are qualitatively similar to ours. 
with $S$ and $P$ waves included is

$$
h=h_{0}(s)+q^{2} h_{1}(s) \cos \Theta,
$$

which can be written, according to (2.7), as

$$
h(s, t)=h_{0}(s)+q^{2} h_{1}(s)\left(1+t / 2 q^{2}\right) .
$$

Because $h$ is a finite polynomial in $t$, it has the property that $h \rightarrow \infty$ as $t \rightarrow \infty$ for $s \neq 0$ [in our particular case $\left.h \sim h_{1}(s) t / 2\right]$. It would then appear to be a restriction on $h$ to make

$$
\lim _{t \rightarrow \infty} h(s=0)
$$

finite; therefore we assume that this is infinite as well. Now the relation

$$
h_{1}=\frac{1}{q^{2}} \int_{-1}^{1} d(\cos \Theta) h \cos \Theta,
$$

can be rewritten, using (2.7), as

$$
h_{1}=\frac{1}{q^{2}} \int_{-4 q^{2}}^{0} \frac{d t}{2 q^{2}} h(s, t)\left(1+\frac{t}{2 q^{2}}\right) \text {. }
$$

We were originally concerned about the factor $q^{-2}$ going to zero as $s$ approaches zero. However, if we put in an expression for $h(s, t)$ which has the property indicated above,

$$
h(s, t) \sim t,
$$

then the factor of $q^{-2}$ in front actually is canceled. To see this we note that as $s \rightarrow 0$, the range of integration goes from $\sim-s^{-1}$ to 0 . The factor $\left(1+t / 2 q^{2}\right)$ approaches -1 , and $q^{-2} \sim s$. This gives us

$$
h_{1} \sim-s \int_{-1 / s}^{0}(s d t) t \sim \text { constant }
$$

The factor of $s$ in front has thus disappeared and there is no a priori reason to believe that the constant is zero.

Another argument starts from the fact that Eq. (2.9) for $q^{2}$ reduces to

$$
q^{2}=\left(W^{2}-4 M^{2}\right) / 4,
$$

when the masses are equal. In this case $q^{2}$ is nonzero at $W^{2}=0$, and there is no reason why (A.1) should vanish at $W^{2}=0$. If the transition from the equal-mass case is smooth, (A.1) should not vanish for the nonequal-mass case either.

\section{APPENDIX B : $N / D$ SOLUTION}

In this appendix we present the details of our $N / D$ solution to the problem of a given set of poles on the dynamic cuts and unitarity on the physical cuts. We write

$$
g=N / D,
$$

and following the procedure outlined in the text we find

$$
\begin{gathered}
N(W)=\sum_{i} \frac{R_{i} D\left(W_{i}\right)}{W-W_{i}} \\
D(W)=1-\frac{W-M}{\pi} \int_{M+\mu}^{\infty} \frac{d W^{\prime}}{W^{\prime 2}} q^{\prime 3}\left[\sum_{i} R_{i} D\left(W_{i}\right)\right. \\
\times\left(\frac{1}{\left(W^{\prime}-W\right)\left(W^{\prime}-M\right)\left(W^{\prime}-W_{i}\right)}\right. \\
\left.\left.-\frac{1}{\left(W^{\prime}+W\right)\left(W^{\prime}+M\right)\left(W^{\prime}+W_{i}\right)}\right)\right] .
\end{gathered}
$$

Now

$$
q^{\prime 2}=\frac{1}{4 W^{\prime 2}}\left[\left(W^{\prime 2}-M^{2}-\mu^{2}\right)^{2}-4 M^{2} \mu^{2}\right],
$$

So that $q^{\prime 3}$ is a complicated function. We can approximate $q^{\prime 3}$ sufficiently well for our purpose by

$$
q^{\prime 3}=\frac{\left(W^{\prime 2}-M^{2}\right)^{3}}{8 W^{\prime 3}}\left[1-3 \mu^{2} \frac{\left(W^{\prime 2}+M^{2}\right)}{\left(W^{\prime 2}-M^{2}\right)^{2}}\right]
$$

We therefore can write

$$
\begin{aligned}
& D(W)=1-\frac{W-M}{8 \pi M} \sum_{i} D\left(W_{i}\right) R_{i} \\
& \times\left[I\left(\omega, \omega_{i}\right)-\frac{3 \mu^{2}}{M^{2}} J\left(\omega, \omega_{i}\right)\right] \\
& I\left(\omega, \omega_{i}\right)=\int_{l}^{\infty} \frac{d \omega^{\prime}}{\omega^{\prime 5}}\left(\omega^{\prime 2}-1\right)^{3}\left[\frac{1}{\left(\omega^{\prime}-\omega\right)\left(\omega^{\prime}-1\right)\left(\omega^{\prime}-\omega_{i}\right)}\right. \\
& J\left(\omega, \omega_{i}\right)=\int_{l}^{\infty} \frac{d \omega^{\prime}}{\omega^{\prime 5}}\left(\omega^{\prime 2}-1\right)\left(\omega^{\prime 2}+1\right) \\
& \quad \times\left[\frac{1}{\left(\omega^{\prime}+\omega\right)\left(\omega^{\prime}+1\right)\left(\omega^{\prime}+\omega_{i}\right)}\right] \\
& {\left[\frac{1}{\left(\omega^{\prime}+\omega\right)\left(\omega^{\prime}+1\right)\left(\omega^{\prime}+\omega_{i}\right)}\right] }
\end{aligned}
$$

where we have introduced $\omega \equiv W / M$ and $l=1+\mu / M$. The integrals are elementary and the results are most conveniently expressed in the form

$$
\begin{gathered}
I\left(\omega, \omega_{i}\right)=\left[1 /\left(\omega-\omega_{i}\right)\right]\left[H(\omega)-H\left(\omega_{i}\right)\right], \\
J\left(\omega, \omega_{i}\right)=\left[1 /\left(\omega-\omega_{i}\right)\right]\left[C(\omega)-C\left(\omega_{i}\right)\right],
\end{gathered}
$$


where

$$
\begin{gathered}
H(\omega)=\omega(1+\omega)\left[-\frac{\left(\omega^{2}-1\right)^{2}}{\omega^{6}} \ln \left[\frac{l^{2}-\omega^{2}}{l^{2}}\right]\right. \\
\left.+\frac{2 \omega^{2}-1}{\omega^{4}}\left(\frac{1}{l^{2}}\right)-\frac{1}{2 \omega^{2}}\left(\frac{1}{l^{4}}\right)\right], \\
C(\omega)=-\omega(1+\omega)\left[\frac{\omega^{2}+1}{\omega^{6}} \ln \left[\frac{l^{2}-\omega^{2}}{l^{2}}\right]\right. \\
\left.+\frac{\omega^{2}+1}{\omega^{4}}\left(\frac{1}{l^{2}}\right)+\frac{1}{2 \omega^{2}}\left(\frac{1}{l^{4}}\right)\right], \\
I(\omega, \omega)=d H(\omega) / d \omega, \\
J(\omega, \omega)=d C(\omega) / d \omega .
\end{gathered}
$$

If we let

$$
\begin{aligned}
& D(2.12 M i)=x+i y, \\
& D(4.32 M i)=u+i v,
\end{aligned}
$$

then upon evaluating $D(\omega)$ at these two points we find the following 4 linear equations

$$
T=\frac{3}{2}\left\{\begin{aligned}
x+i y= & 1.312-0.072 i+x(0.491) \\
& +y(-0.295+0.354 i) \\
& +u(0.437-0.596 i)+v(0.398) \\
u+i v= & 1.49-0.075 i+x(1.01) \\
& +y(-0.295+0.427 i) \\
& +u(0.444-0.796 i)+v(0.898)
\end{aligned}\right.
$$

whose solutions are those given in the text (Eq. 6.26). For the other isotopic spin state we find

$$
T=\frac{1}{2}\left\{\begin{array}{c}
x+i y=0.844+0.036 i+x(-0.246) \\
+y(0.148-0.177 i) \\
+u(-0.219+0.298 i)+v(-0.199) \\
u+i v=0.755+0.037 i+x(-0.505) \\
+y(0.148-0.213 i) \\
+u(-0.222+0.398 i)+v(-0.449)
\end{array}\right.
$$

whose solutions are also given in the text (Eq. 6.27). In order to calculate $\operatorname{Re} D(\omega)$ along the right cut, we must evaluate terms of the form

$$
\begin{aligned}
& \operatorname{Re}\left[\frac{(r+i s)}{\omega-(\alpha+i \beta)}[(X+i Y)-(U+i V)]\right. \\
& \left.\quad+\frac{(\gamma-i s)}{\omega-(\alpha-i \beta)}[(X+i \dot{Y})-(U-i V)]\right]
\end{aligned}
$$

where

$$
\begin{aligned}
R_{i} D\left(W_{i}\right) & =r+i s, \\
H(\omega)-\left(3 \mu^{2} / M^{2}\right) C(\omega) & =X+i Y, \\
H\left(\omega_{i}\right)-\left(3 \mu^{2} / M^{2}\right) C\left(\omega_{i}\right) & =U+i V, \\
\omega_{i} & =\alpha+i \beta .
\end{aligned}
$$

The above expression reduces to

$$
\begin{aligned}
& r\left\{\frac{2}{(\omega-\alpha)^{2}+\beta^{2}}[(\omega-\alpha)(X-U)+\beta V]\right\} \\
& +s\left\{\frac{2}{(\omega-\alpha)^{2}+\beta^{2}}[V(\omega-\alpha)-\beta(X-U)]\right\},
\end{aligned}
$$

and the only complicated $\omega$ dependence is in the function

$$
X=\operatorname{Re}\left[H(\omega)-\left(3 \mu^{2} / M^{2}\right) C(\omega)\right] .
$$

Thus, even though the logarithms in $H(\omega)$ and $C(\omega)$ are complex, we only need to know their real parts.

\section{APENDIX C: CONVERGENCE OF THE LEGENDRE EXPANSIONS IN THE CROSSED CHANNELS}

In this appendix we wish to examine the region of convergence of the Legendre expansions in the crossed channels. We shall look at channel III in some detail and then just state the results for channel II as the procedure is exactly the same. Our starting point is the following theorem ${ }^{33}$ : If $F(z)$ is analytic inside an ellipse with foci at +1 , then it can be expanded in a Legendre series $F(z)=\sum_{l} a_{l} P_{l}(z)$ within the ellipse. Our task is to find the singularities in the function $A_{3}{ }^{i}\left(s, t^{\prime}\right)$ in the complex $y$ plane where $y$ is the cosine of the angle of scattering in channel III :

$$
y(t, s)=\frac{s+t / 2-M^{2}-\mu^{2}}{2\left(t / 4-M^{2}\right)^{\frac{1}{2}}\left(t / 4-\mu^{2}\right)^{\frac{1}{2}}} .
$$

We emphasize again that in this channel $t$ is the energy and is real. It is the extension to complex momentum transfer, $s$, in which we are interested.

The singularities in $A_{3}{ }^{i}\left(s, t^{\prime}\right)$ are given to us by Eq. (4.3). We need only investigate the first term in that equation since the second term is obtained from it through the crossing transformation, $s \leftrightarrow u$ at fixed $t$ and we see from our equation for $y(t, s)$ that under this transformation

$$
y(t, s)^{\times}=-y(t, s) .
$$

This means that if we find singularities for positive $y$ from the first term in Eq. (4.3), the second term will give a symmetric singularity at $-y$. We therefore consider

$$
\frac{1}{\pi} \int_{(M+\mu)^{2}}^{\infty} \frac{\rho_{13}{ }^{i}\left(s^{\prime}, t^{\prime}\right) d s^{\prime}}{s^{\prime}-s} .
$$

Let the smallest value of $s$ for which the denominator can vanish be $s_{\text {B.c. }}(t)$. (See reference 8 and Fig. 2.) We note that the denominator vanishes only for real $s$.

${ }^{33}$ E. T. Whittaker and G. N. Watson, $A$ Course of Modern Analysis (The Macmillan Company, New York, 1943), p. 322. 


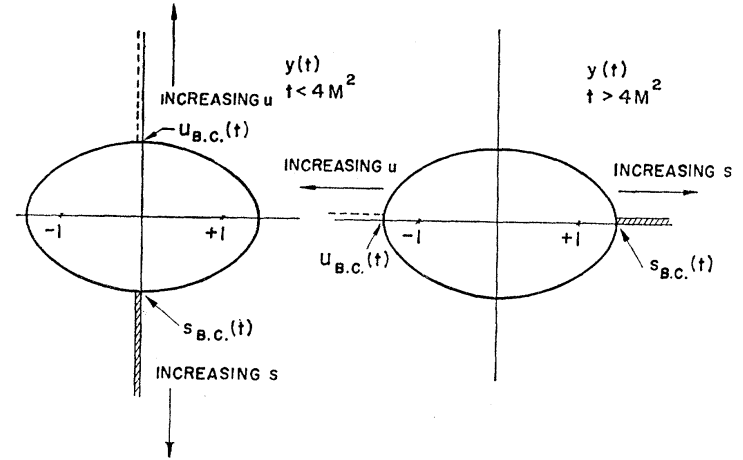

FIG. 15. Ellipses of convergence of the Legendre expansion in channel III. The cosine of the production angle is denoted by $y[\mathrm{Eq} .(2.34)]$.

We split the $t$ region of interest into two parts,

(1) $4 \mu^{2} \leqslant t \leqslant 4 M^{2}, \quad y(t, s)=-i y_{0}$,

(2) $t \geqslant 4 M^{2}$

$$
y_{0}=\frac{s+t / 2-M^{2}-\mu^{2}}{2\left(M^{2}-t / 4\right)^{\frac{1}{2}}\left(t / 4-\mu^{2}\right)^{\frac{1}{2}}}
$$

$$
y(t, s)=\frac{s+t / 2-M^{2}-\mu^{2}}{2\left(t / 4-M^{2}\right)^{\frac{1}{2}}\left(t / 4-\mu^{2}\right)^{\frac{1}{2}}} .
$$

Since the denominator of (C.3) vanishes for real $s$ only, we find that for region 1 the corresponding $y$ is imaginary whereas for region 2 it is real. The two cases are indicated in Fig. 15.

We now have the ellipse, in the complex $y$ plane, inside of which the Legendre expansion converges. The corresponding region in the complex $s$ plane is determined by Eqs. (C.1) and (C.4). In order to get a very simple upper limit to the region of convergence for $s$ lying on the circle, we can imagine our region in the $y$ plane to be an infinite strip instead of an ellipse. In the first case, the condition that $y$ be inside the strip is

$$
|\operatorname{Im} y| \leqslant \frac{S_{\text {B.C. }}(t)+t / 2-M^{2}-\mu^{2}}{2\left(M^{2}-t / 4\right)^{\frac{1}{2}}\left(t / 4-\mu^{2}\right)^{\frac{1}{2}}} .
$$

In the second case the condition is

$$
|\operatorname{Re} y| \leqslant \frac{S_{\text {B.C. }}(t)+t / 2-M^{2}-\mu^{2}}{2\left(t / 4-M^{2}\right)^{\frac{\lambda}{2}}\left(t / 4-\mu^{2}\right)^{\frac{1}{2}}} .
$$

If we now let $s$ become complex in Eqs. (C.5) and (C.6), then both conditions reduce to

$$
\left|\operatorname{Re}(s)+t / 2-M^{2}-\mu^{2}\right| \leqslant s_{\text {B.C. }}(t)+t / 2-M^{2}-\mu^{2} .
$$

We investigate two possibilities.

\section{A. $W$ on the Real Axis}

In this case $\left|W^{2}+t / 2-M^{2}-\mu^{2}\right| \leqslant s_{\text {B.c. }}(t)+t / 2-M^{2}$ $-\mu^{2}$ implies $W^{2} \leqslant s_{\text {B.c. }}(t)$. Since $t$ goes over all values $4 \mu^{2} \leqslant t \leqslant \infty$ in (C.7), we must take $s_{\text {B.C. }}(t)_{\min }=(M+\mu)^{2}$. The Legendre series converges for $W$ real and

$$
|W| \leqslant(M+\mu) \text {. }
$$

\section{B. $W$ on the $\pi-\pi$ Circle}

In this case we write $W^{2}=\left(M^{2}-\mu^{2}\right) e^{2 i \chi}$ and our condition becomes

$$
\begin{aligned}
\left|2 \mu^{2}+2\left(M^{2}-\mu^{2}\right) \sin ^{2} \chi-t / 2\right| & \\
& \leqslant s_{\text {B.C. }}(t)+t / 2-M^{2}-\mu^{2} .
\end{aligned}
$$

Assuming the left-hand side is positive (the converse inequality is always true) we find

$$
\sin ^{2} \chi \leqslant \frac{s_{\text {B.C. }}(t)+t-M^{2}-3 \mu^{2}}{2\left(M^{2}-\mu^{2}\right)} .
$$

From reference 8 we have $\left[s_{\text {B.c. }}(t)+t\right]_{\min } \simeq 96 \mu^{2}$ for $t \simeq 12 \mu^{2}$ and $t \simeq 24 \mu^{2}$. This gives

$$
\begin{aligned}
\sin ^{2} \chi & \leqslant 0.52, \\
0 & \leqslant|\chi| \leqslant 46^{\circ}, \\
134^{\circ} & \leqslant|\chi| \leqslant 180^{\circ} .
\end{aligned}
$$

For these values of $\chi$ and $t$ the left-hand side of (C.9) was positive as assumed.

A numerical calculation of the correct elliptical region of convergence has been carried out by Frazer and Fulco. ${ }^{5}$ They find that the series converges if

$$
\begin{aligned}
0 & \leqslant|\chi| \leqslant 33^{\circ}, \\
147^{\circ} & \leqslant|\chi| \leqslant 180^{\circ} .
\end{aligned}
$$

A similar treatment of channel II shows that the channel II scattering amplitude along the real axis between $-M-\mu$ and $M+\mu$ can be expanded in Legendre polynomials for any $u>(M+\mu)^{2} .{ }^{34}$ The amplitude for any particular finite $u>(M+\mu)^{2}$ converges over a somewhat wider range of $W$.

The results of this appendix are indicated in Fig. 5.

${ }^{34}$ W. R. Frazer (private communication) has shown that the region of convergence of the Legendre expansion extends for a short distance along the imaginary axis near $W=0$. 